\title{
Neutron spin echo investigation of the concentration fluctuation dynamics in melts of diblock copolymers
}

H. Montes, M. Monkenbusch, L. Willner, S. Rathgeber, L. Fetters, and D. Richter

Citation: The Journal of Chemical Physics 110, 10188 (1999); doi: 10.1063/1.478891

View online: https://doi.org/10.1063/1.478891

View Table of Contents: http://aip.scitation.org/toc/jcp/110/20

Published by the American Institute of Physics

\section{Articles you may be interested in}

Polymer dynamics under cylindrical confinement featuring a locally repulsive surface: A quasielastic neutron scattering study

The Journal of Chemical Physics 146, 203306 (2017); 10.1063/1.4974836

Perspective: Outstanding theoretical questions in polymer-nanoparticle hybrids

The Journal of Chemical Physics 147, 020901 (2017); 10.1063/1.4990501

A Theory of the Linear Viscoelastic Properties of Dilute Solutions of Coiling Polymers

The Journal of Chemical Physics 21, 1272 (1953); 10.1063/1.1699180

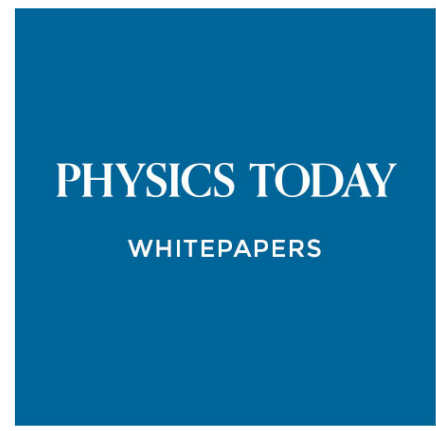




\title{
Neutron spin echo investigation of the concentration fluctuation dynamics in melts of diblock copolymers
}

\author{
H. Montes \\ ESPCI, Physico-Chimie Structurale et Macromoleculaire, 10-Rue Vauquelin, 75005 Paris Cedex, France \\ M. Monkenbusch and L. Willner \\ Institut für Festkörperforschung, Forschungszentrum Jülich GmbH, 52425 Jülich, Germany \\ S. Rathgeber \\ National Institute of Standards and Technology, Gaithersburg, Maryland 20899 \\ L. Fetters \\ Exxon, Research and Engineering Company, Corporate Research Laboratories, Annandale, \\ New Jersey 08801 \\ D. Richter \\ Institut für Festkörperforschung, Forschungszentrum Jülich GmbH, 52425 Jülich, Germany
}

(Received 2 December 1998; accepted 4 March 1999)

\begin{abstract}
Diblock copolymers in the melt exhibit order-disorder phase transitions (ODT), which are accompanied by strong concentration fluctuations. These transitions are generally described in terms of the random phase approximation (RPA) of Leibler and Fredrickson, which is able to explain small angle scattering results in the neighborhood of the ODT, in particular around the correlation peak at $q^{*}$. The RPA theory has been extended to include dynamical phenomena, predicting the short time relaxation of the dynamic structure factor in polymeric multicomponent systems. We report small angle neutron scattering and neutron spin echo experiments on polyethyleneblock-polyethylethylene (PE-PEE) and poly(ethylene-propylene)-block-polyethylethylene (PEP-PEE) copolymers with molecular weights of 16.500 and $68.000 \mathrm{~g} / \mathrm{mol}$, which explore the structure and dynamics of these block copolymers. Studying melts with different hydrogen/ deuterium labeling it was possible to observe experimentally the different relaxation modes of such systems separately. In particular the collective relaxation behavior as well as the single chain motion were accessed. The experimental results were quantitatively compared with the RPA predictions, which were based solely on the dynamical properties of the corresponding homopolymers and the static structure factors. The collective dynamics exhibits an unanticipated fast relaxation mode. This mode is most visible at low wave numbers $\left(q \geqslant q^{*}\right)$ but extends to length scales considerably shorter than the radius of gyration. Furthermore, the dynamical RPA yields expressions for the mobilities of chain segments in the block copolymer melt. These combination rules are at variance with the experimental findings for the single chain dynamics, while they hold for the collective response. (C) 1999 American Institute of Physics. [S0021-9606(99)52120-X]
\end{abstract}

\section{INTRODUCTION}

Diblock copolymers represent an important and interesting class of polymeric materials which are studied presently by quite a large number of research groups. Up to now, most of the scientific interest has been devoted to static properties and to the identification of the relevant parameters controlling thermodynamical properties and thus morphologies. ${ }^{1-4}$ All these studies have allowed for improvements to the random phase approximation (RPA) theory first developed by Leibler. ${ }^{5}$ In particular, the role of the concentration fluctuations which occur and accompany the order-disorder transition is studied. ${ }^{6-8}$

These concentration fluctuations which are pivotal to the phase transitions in block copolymer melts are dynamic in nature. They lead to a renormalization of the relevant interaction parameters and are thought to be responsible for the induction of the first-order nature of the phase transition. ${ }^{6-10}$
Such fluctuations are better studied in dynamic experiments. Thus, recently one observes an increasing interest in diblock copolymer dynamics. These dynamical properties are analyzed through experimental, theoretical, ${ }^{11,12}$ and computer simulation approaches ${ }^{13,14}$ with the aim of determining the main features of diblock copolymer dynamics in comparison to homopolymer dynamics. There are three main issues.

(1) The first issue concerns the relation between the dynamics of a diblock copolymer and that of the homopolymers composing the diblock chains. Is it possible to understand the single chain and collective dynamics of A-B diblock copolymer chains from the dynamics of the homopolymers A and B?

(2) The second main point concerns the concentration fluctuations and the influence of the order-disorder transition (ODT) on these fluctuations.

(3) The third relates to the dynamics of the chain segments 
located close to the interface between the blocks. Is there some particular interface dynamics?

By analogy with the RPA describing the static properties, a dynamic RPA theory was developed. ${ }^{15}$ This theory describes the response functions of composite polymer systems using the single chain dynamics of the corresponding homopolymers and the static structure factors as input. The main prediction concerns the dynamics of concentration fluctuations (collective dynamics) which display a critical slowing down around wave numbers $q^{*} \sim 1 / R_{g}$ in the neighborhood of the ODT, $R_{g}$ being the radius of gyration.

Most of experiments made on block copolymer dynamics have been designed to test the validity and the limits of this dynamic RPA approach. ${ }^{16,17}$ They have investigated the diffusive motions of the block copolymer chains, which occur at length scales larger than the radius of gyration ( $q$ $<1 / R_{g}$ ). These experiments were performed using light scattering $^{18-21}$ and photon correlation spectroscopy; ${ }^{22}$ most of them were made on block copolymers in solution since melts are generally beyond the scope of dynamic light scattering experiments. Furthermore, such techniques do not allow to observe the single chain dynamics in a melt. There are also experiments dealing with the reptation motions in block copolymers. $^{23,24}$

In this work, we approach two of the three issues concerning block copolymer dynamics. We present investigations on the dynamics of diblock copolymer melts at length scales smaller than the radius of gyration of the chains ( $q$ $>1 / R_{g}$ ) and at a time scale where entanglement effects are negligible. Both the single chain and collective dynamics are analyzed and compared to the dynamics of the corresponding homopolymers. A quantitative comparison of the experimental results with the RPA predictions is made. Finally, the influence of the concentration fluctuations is analyzed. A comparatively fast relaxation process is observed at a length scale where the RPA theory predicts a slowing down of the concentration fluctuations.

This article is arranged as follows: The main features of the static and dynamic RPA theory are presented in Sec. II. Section III reports the results obtained by small angle neutron scattering (SANS) and neutron spin echo spectroscopy which are compared to the RPA predictions in Sec. IV. The difference between single chain and collective dynamics in relation with the theoretical predictions are discussed in Sec. V. Finally the concluding remarks constitute Sec. VI.

\section{THEORY}

In this section, we present the multicomponent random phase approximation (RPA) approach developed by Akcasu et al. ${ }^{15}$ for the calculation of $S(q, t)$ of incompressible diblock copolymer mixtures. The theoretical foundations for the calculations of $S(q, t)$ for a single chain in the Rouse model are briefly recalled.

\section{A. Multicomponent random phase approximation}

We consider an incompressible $(m+1)$ multicomponent mixture of polymers consisting of $m$ different types of polymer chains within a matrix referred to as " $o$." Components thereby may be either homopolymers of a given chemical species or, e.g., homopolymer sections in block copolymers. Hydrogenated and deuterated species of the same homopolymer are considered as different components. In this context a diblock copolymer is a two-component polymer system. A mixture of partially protonated diblock chains $\mathrm{hA}-\mathrm{dB}$ with deuterated diblock chains consequently is regarded as a fourcomponent system. The present work concerns such fourcomponent systems.

We denote the fluctuations of the number density of the monomers of component $j$ at a point $\underline{r}$ and at a time $t$ as $\rho_{j}(\underline{r}, t)$. With this definition we have $\left\langle\rho_{j}(\underline{r}, t)\right\rangle=0$.

Now we introduce the Hamiltonian of the system $H$ and add an external potential $V(t)$,

$$
\underline{V}(t)=\int d^{3} \underline{r} \varrho^{+}(\underline{r}) U(\underline{r}, t),
$$

where $\varrho(\underline{r})=\operatorname{col}\left[\rho_{1}(\underline{r}), \rho_{2}(\underline{r}), \ldots, \rho_{m}(r), \rho_{o}(\underline{r})\right], \varrho^{+}(\underline{r})$ is the Hermitian conjugate of $\varrho(\underline{r})$ and $U(A \underline{r}, t)$ is an external time-dependent potential coupled to the density of the monomers.

In the linear response theory ${ }^{25}$ the Fourier-Laplace transform of the mean of the number density $\bar{\rho}(r, s)$ is expressed as

$$
\bar{\varrho}(r, s)=-\underline{\chi}(q, s) \underline{U}(q, s),
$$

where $\underline{\chi}(q, s)$ is the $(m+1) *(m+1)$ dynamic response matrix. In order to proceed further we split the Hamiltonian into two parts, $H=H_{0}+H_{1} . H_{0}$ is the Hamiltonian of the bare system. In this reference system the interactions among the monomers are removed but the chain connectivity is preserved. $H_{1}$ is the part of the Hamiltonian that describes the interactions $W_{i j}(r)$ between monomers in the system and the incompressibility constraints. These are taken into account by a Lagrange multiplier $u(r, t)$ which may be considered as an external potential which couples to all monomers equally such that the perturbed average of the total density is zero.

For $H_{1}$ results

$H_{1}=\int d^{3} \underline{r} \varrho(\underline{r}) \underline{u}(\underline{r}, t)+\int d^{3} \underline{r} \underline{\varrho}^{+}(\underline{r}) \int d r^{\prime} \underline{\underline{W}}(\underline{r}-\underline{r}) \bar{\varrho}\left(\underline{r}^{\prime}\right)$.

We treat $H_{1}$ as perturbation, insert into Eq. (2), and obtain

$\bar{\varrho}(q, s)=-\underline{\underline{\chi}}^{0}(q, s)[\underline{U}(q, s)+u(q, s) \underline{E}+\underline{\underline{W}}(q, s) \bar{\varrho}(q, s)$,

with the boundary condition $\underline{E}^{T} \bar{\varrho}(r, s)=0$, where $\underline{E}$ is a column matrix with all elements $=1$.

Now one may either eliminate $u(q, s)$ directly using the boundary condition $E^{T} \bar{\rho}(r, s)=0$, or one may use the matrix component " 0 " in order to fulfill the incompressibility constraint. For that purpose we have to assume that " 0 ", is not coupled to other components in the bare system $\left(\chi_{0 i}^{0}=0\right.$ for $i \neq 0)$. Then $u(q, s)$ can be expressed in terms of the matrix response

$$
u(q, s)=\frac{-\bar{\rho}_{0}(q, s)}{\chi_{00}^{0}(q, s)}-U_{0}(q, s)-\sum_{j=1}^{m} W_{0 j}(q) \bar{\rho}_{j}(q, s) .
$$

Substituting $u(q, s)$ into Eq. (4) we find 


$$
\bar{\varrho}(q, s)=-\underline{\underline{\chi}}^{0}(q, s)\left[\underline{U}(q, s)+k_{B} T \underline{\underline{\nu}}(q, s) \bar{\varrho}(q, s)\right] .
$$

The excluded volume matrix defined by Eq. (6) has the following elements:

$$
\begin{aligned}
& \nu_{i i}(q, s)=\frac{1}{k_{B} T \chi_{00}^{0}(q, s)}-2 \kappa_{i 0}(q), \\
& \nu_{i j}(q, s)=\frac{1}{k_{B} T \chi_{00}^{0}(q, s)}+\kappa_{i j}(q)-\kappa_{i 0}(q)-\kappa_{j 0}(q),
\end{aligned}
$$

where

$$
\kappa_{i j}(q)=\frac{1}{k_{B} T}\left[W_{i j}(q)-\frac{1}{2}\left[W_{i i}(q)-W_{j j}(q)\right]\right] .
$$

$\kappa_{i j}$ is the Flory-Huggins interaction parameter between the $i$ and $j$ monomers. In Eq. (6), the matrices have a dimension $(m)^{*}(m)$. We note that the $s$ dependence of the excluded volume matrix is solely determined by the contribution of the bare susceptibility $\chi_{00}^{0}(q, s)$ from the matrix component. Finally, combining Eq. (6) with Eq. (2) the response function in the interacting system is given by

$$
\underline{\chi}(q, s)=\left[\underline{\underline{I}}+k T \underline{\underline{\chi}}^{0}(q, s) \underline{\underline{\nu}}(q, s)\right]^{-1} \underline{\underline{\chi}}^{0}(q, s),
$$

where $\underline{\underline{I}}$ is the unit matrix. For $s=0$, we obtain the static response function $\underline{\chi}(q)$,

$$
\underline{\chi}(q)=\left[\underline{\underline{I}}+k T \underline{\underline{\chi}}^{0}(q) \underline{\underline{\nu}}(q)\right]^{-1} \underline{\underline{\chi}}^{0}(q)
$$

or

$$
\frac{1}{\underline{\chi}(q)}=\frac{1}{\underline{\chi}_{0}(q)}+k T \underline{\underline{\nu}}(q) .
$$

Equations (8) and (9) are the basic results needed in order to calculate the static and dynamic structure factors $\underline{\underline{S}}(q)$ and $\stackrel{S}{S}(q, t)$, respectively.

\section{B. Static scattering function}

According to the linear response theory, ${ }^{25}$ the static scattering function $S(q)$ relates to the static response function $\underline{\chi}(q)$,

$$
\underline{\chi}(q)=\frac{1}{k_{B} T V} \underline{\underline{S}}(q),
$$

with $V$ the volume of the system. This relation also holds between $\underline{\chi}^{0}(q)$ and $\underline{\underline{S}}^{0}(q)$. From Eq. (9) the basic result of RPA for the static structure factor matrix immediately follows:

$$
\frac{1}{\underline{\underline{S}}(q)}=\frac{1}{\underline{\underline{S}}_{0}(q)}+\underline{\underline{\nu}}(q) .
$$

For an A-B diblock copolymer system, Eq. (11) yields for the partial structure factors $S_{i j}(q)$,

$$
\begin{aligned}
S_{a a}(q) & =S_{b b} \\
& =-S_{a b}=\frac{\left(S_{a a}^{0} S_{b b}^{0}-S_{a b}^{0^{2}}\right)}{S_{a a}^{0}+S_{b b}^{0}+2 S_{a b}^{0}-2 \kappa\left(S_{a a}^{0} S_{b b}^{0}-S_{a b}^{0^{2}}\right)} .
\end{aligned}
$$

For the limit of long chains and equal segment lengths within the two blocks, the bare static structure factors $S_{i j}^{0}(q)$ can be expressed in terms of Debye functions $g(f, x)$,

$$
\begin{aligned}
& g(f, x)=\frac{2}{f^{2} x^{2}}[f x+\exp (-f x)-1], \\
& x=q^{2} R_{g}^{2},
\end{aligned}
$$

with $R_{g}$ denoting the radius of gyration of the diblock chain and $f$ the volume fraction of block $\mathrm{A}$,

$$
\begin{aligned}
S_{11}^{0}= & \phi_{1} N f g(f, x), \\
S_{22}^{0}= & \phi_{2} N(1-f) g(1-f, x), \\
S_{12}^{0}= & \frac{1}{2} \frac{\sqrt{\phi_{1} \phi_{2} f(1-f) N^{2}}}{f(1-f)}\left(g(1, x)-f^{2} g(f, x)\right. \\
& \left.-(1-f)^{2} g(1-f, x)\right) .
\end{aligned}
$$

Using the notions $\Delta=S_{11}^{0} S_{22}^{0}-\left(S_{12}^{0}\right)^{2}$ and $\Sigma=S_{11}^{0}+S_{22}^{0}$ $+2 S_{12}^{0}$ we finally arrive at the well-known Leibler equation of the static structure factor:

$$
S(q)=\frac{\Delta}{\sum-2 \kappa \Delta} .
$$

\section{Dynamic structure factor}

In the frame of linear response theory the dynamic response function matrix $\underline{\chi}(q, t)$ is related to the intermediate dynamic structure factor by

$$
k_{B} T \underline{\underline{\chi}}(q, t)=-\frac{\partial}{\partial t} \underline{S}(q, t)
$$

The Laplace transformation yields

$$
\underline{\underline{\chi}}(q, s)=-\frac{\beta}{V}[s \underline{\underline{S}}(q, s)-\underline{\underline{S}}(q)] .
$$

In order to proceed, we have to connect to results from diffusion theories based on the generalized Langevin equation. From there the Laplace transformed dynamic structure factor may be written as

$$
\underline{\underline{S}}(q, s)=\frac{1}{s \underline{\underline{I}}+q^{2} \underline{\underline{D}}(q, s)} \underline{\underline{S}}(q) .
$$

$\underset{\equiv}{D}(q, s)$, thereby, stands for a $q$ - and $s$-dependent generalized diffusion matrix:

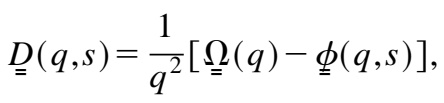

where $\underline{\underline{\Omega}}=\lim _{t \rightarrow 0}[\partial \underline{\underline{S}}(q, t) / \partial t] \underline{\underline{S}}^{-1}(q)$ is the so-called "ffirst cumulant" matrix and $\Phi(q, s)$ a Laplace transformed memory function, which we will neglect in the following.

With the use of Eq. (18) we may rewrite Eq. (17) and arrive at

$$
\underline{\underline{\chi}}(q, s)=\frac{\beta}{V} q^{2} \underline{\underline{D}}(q, s) \frac{1}{s \underline{\underline{I}}+q^{2} \underline{\underline{D}}(q, s)} \underline{\underline{S}}(q) .
$$


Equations (20) also holds for $\underline{\underline{\chi}}^{0}(q, s)$. Introducing Eq. (20) both for $\underline{\underline{\chi}}(q, s)$ and $\underline{\underline{x}}^{0}(q, s)$ into Eq. (8) and exploiting Eq. (11), we obtain

$$
\begin{aligned}
\frac{1}{\underline{D}(q, s) \underline{\underline{S}}(q)}= & \frac{1}{\underline{\underline{D}}^{0}(q, s) \underline{\underline{S}}^{0}(q)}+\frac{q^{2} k_{B} T}{V} \\
& \times \frac{1}{S}[\underline{\underline{\nu}}(q, s)-\underline{\underline{\nu}}(q)] .
\end{aligned}
$$

Now we define a generalized mobility matrix $\underline{\underline{\mu}}(q, s)$ $=\left(1 / k_{B} T\right) \underline{\underline{D}}(q, s) \underline{\underline{S}}(q)$ and we furthermore realize that the last term in Eq. (21) is solely determined by the bare response function of the matrix component Eq. (7),

$$
\underline{\underline{\nu}}(q, s)-\underline{\underline{\nu}}(q)=\beta\left[\frac{1}{\chi_{00}^{0}(q, s)}-\frac{1}{\chi_{00}^{0}(q)}\right] \underline{\underline{E E^{T}}},
$$

with $\underline{\underline{E E^{T}}}$ being a matrix containing 1 at all positions. Now we apply Eqs. (16)-(18) to the dynamics of the bare matrix and arrive at a relation between the bare response function $\chi_{00}^{0}(q, s)$ and the corresponding generalized mobility,

$$
\frac{1}{\chi_{00}^{0}(q, s)}=V\left[\frac{1}{q^{2}} \frac{s}{\mu_{00}^{0}(q, s)}+\frac{1}{\beta S_{00}^{0}(q)}\right] .
$$

Using $\chi_{00}^{0}(q)=(\beta / V) S_{00}^{0}(q)$ and inserting Eq. (23) into Eq. (22) yields

$$
[\underline{\underline{\nu}}(q, s)-\underline{\underline{\nu}}(q)]=\frac{V}{q^{2} k T} \frac{s}{\mu_{00}^{0}(q, s)} \underline{\underline{E}} \underline{E}^{T} .
$$

With Eq. (24) substituted into Eq. (21) we finally arrive at a RPA equation for the generalized mobility,

$$
\frac{1}{\underline{\underline{\mu}}(q, s)}=\frac{1}{\underline{\underline{\mu}}^{0}(q, s)}+\frac{1}{\mu_{00}^{0}(q, s)} \underline{\underline{E}}^{T} \text {. }
$$

With the aid of the Sherman-Morrison formula, Akcasu and Tombakoglu have inverted Eq. (25) with the result

$$
\underline{\underline{\mu}}(q, s)=\underline{\underline{\mu}}^{0}(q, s)-\frac{\underline{\underline{\mu}}^{0}(q, s) \underline{\underline{E}}^{T} \underline{\underline{\mu}}^{0}(q, s)}{\mu_{00}^{0}(q, s)+\underline{\underline{E}}^{T} \underline{\underline{\mu}}^{0}(q, s) \underline{E}} .
$$

We note that the first cumulant matrix of Eq. (19) relates to $\mu(q, s)$ by

$$
\underline{\underline{\Omega}}(q)=k T q^{2} \underline{\underline{\mu}}(q, s \rightarrow \infty) \underline{\underline{S}}(q)^{-1} .
$$

Furthermore, since $\underline{\underline{\mu}}(q, s)$ only depends on bare mobilities, the interaction terms expressed in terms of the Flory Huggins parameters $\kappa_{i j}$ do not influence the mobility.

For the case of Rouse dynamics, which only depends on local friction of the bare mobility matrix,

$$
\mu_{i j}^{0}(q, s \rightarrow \infty)=m_{i j}(q)=\delta_{i j} \frac{L_{i}}{\zeta_{i}}
$$

is diagonal, $\zeta_{i}$ being the monomeric friction coefficients of component $i$ and $L_{i}$ the number of all monomers of this component in the system. If we normalize to the total number of all monomers and consider proper different monomeric volumes, the resulting mobility matrix elements are $m_{i j}=\left(\phi_{i} / \zeta_{i}\right) \delta_{i j}$ where $\phi_{i}$ is the volume fraction of component $i$.

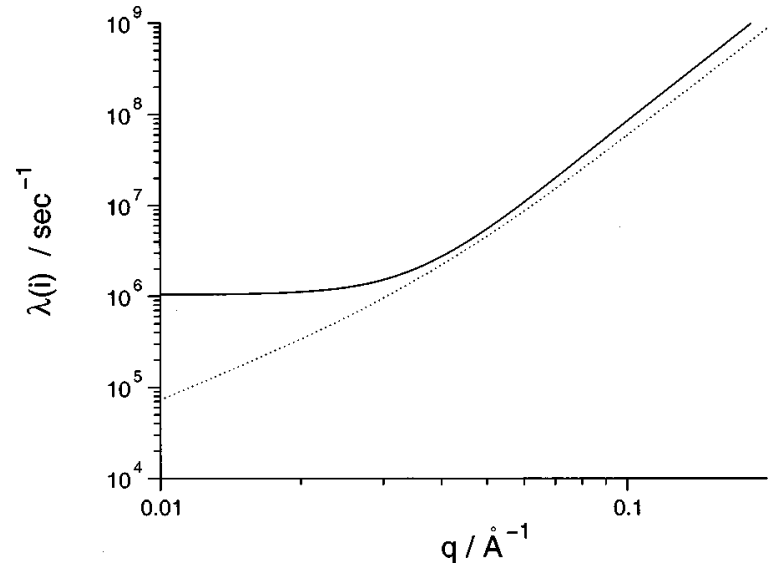

FIG. 1. (a) $Q$ dependence of the two eigenvalues $\Gamma_{1}(q)$ (solid line) and $\Gamma_{2}(q)$ (dotted line) predicted by a two-component dynamic RPA approach for the case of an $\mathrm{hA}-\mathrm{dB}$ labeled diblock copolymer melt. Calculations were performed with $f=0.5, R_{g \mathrm{~A}}=R_{g \mathrm{~B}}=40 \AA, N_{\mathrm{A}}=N_{\mathrm{B}}=200, \kappa_{12}=0$. $\Gamma_{1}(q)$ describes the collective mode of diblock copolymer chains.

For a time-independent mobility or diffusion matrix Eq. (18) may be solved easily with the result

$$
\underline{\underline{S}}(q, t)=\sum_{i} \underline{\alpha}_{i}(q) \underline{\beta}_{i}^{T}(q) e^{-\lambda_{i}(q) t} \underline{\underline{S}}(q),
$$

where $\underline{\alpha}_{i}(q)$ and $\underline{\beta}_{i}(q)$ are the right- and left-hand eigenvectors of the matrix $\Omega$ with respect to the eigenvalues $\lambda_{i}(q)$.

The measured intensity in a scattering experiment depends on the scattering length density contrasts of the different components " $i$ " with the respect to the matrix

$$
K_{i}=\frac{\sum b_{i}}{v_{i}}-\frac{\sum b_{0}}{v_{0}}
$$

where $b_{i}$ are the scattering lengths of the atoms within a monomer of component " $i$ " and $v_{i}$ is the monomer volume, $b_{0}$ and $v_{0}$ denote the same quantities for the matrix. The measured intensity follows as

$$
I(q, t)=\underline{K}^{T} \underline{\underline{S}}(q, t) \underline{\underline{K}},
$$

where $\underline{K}$ is the vector of the component contrast factors $K_{i}$.

Using the component contrast factors we may also give a general result for the first cumulant $\Gamma(q)$ under arbitrary contrast. Using the definition, we have

$$
\Gamma(q)=-\lim _{t \rightarrow 0} \frac{\partial I(q, t)}{\partial t} \frac{1}{I(q)}=\frac{\underline{K}^{T} \underline{\underline{\Omega}} \underline{\underline{S}}(q) \underline{K}}{\underline{K}^{T} \underline{\underline{S}}(q) \underline{K}} .
$$

With Eq. (27) this may also be written as

$$
\Gamma(q)=k_{B} T q^{2} \frac{\underline{K}^{T} \underline{\underline{\mu}}(q, s \rightarrow \infty) \underline{K}}{\underline{K}^{T} \underline{\underline{S}}(q) \underline{K}} .
$$

\section{Results for a diblock copolymer melt and a mixture of two different diblocks}

\section{Diblock copolymer}

The relaxational motion of a diblock copolymer may be expressed in terms of two relaxation modes characterized by the eigenvalues $\Gamma_{1}(q)$ and $\Gamma_{2}(q)$ of the first cumulant matrix $\Omega(q)$ [Eq. (19)]. Figure 1(a) displays these eigenvalues for a 

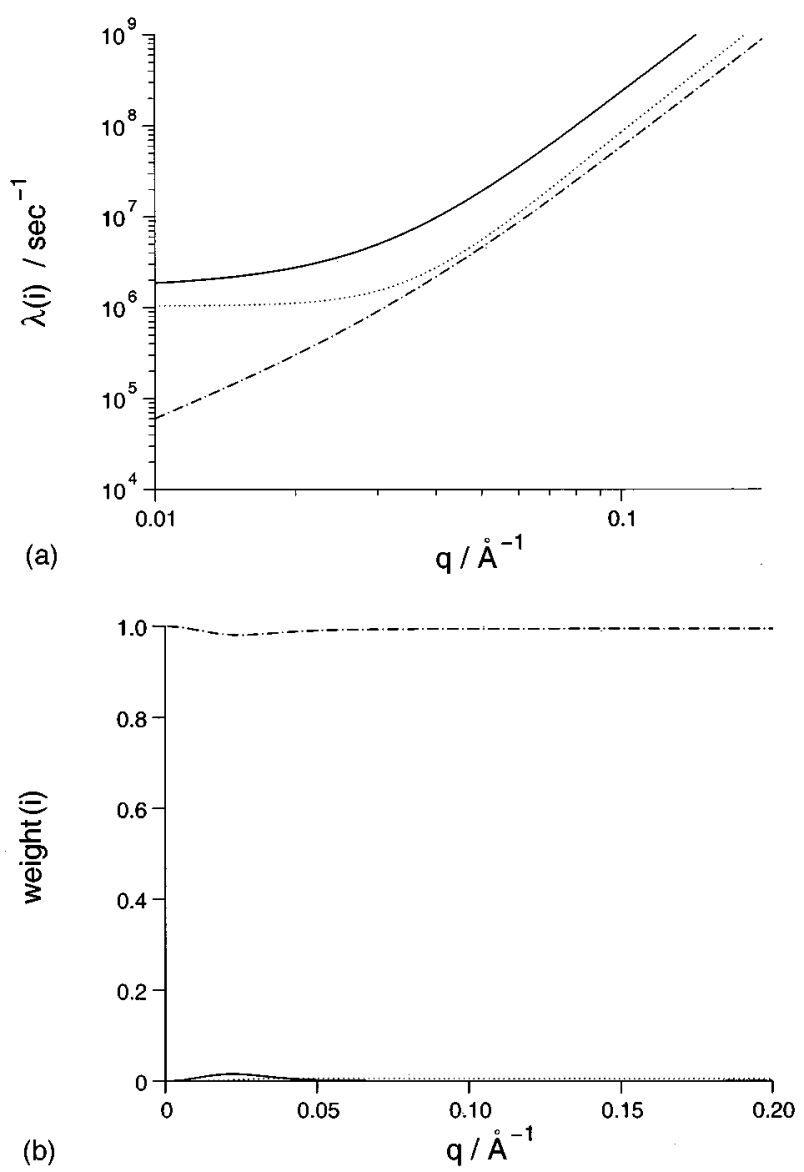
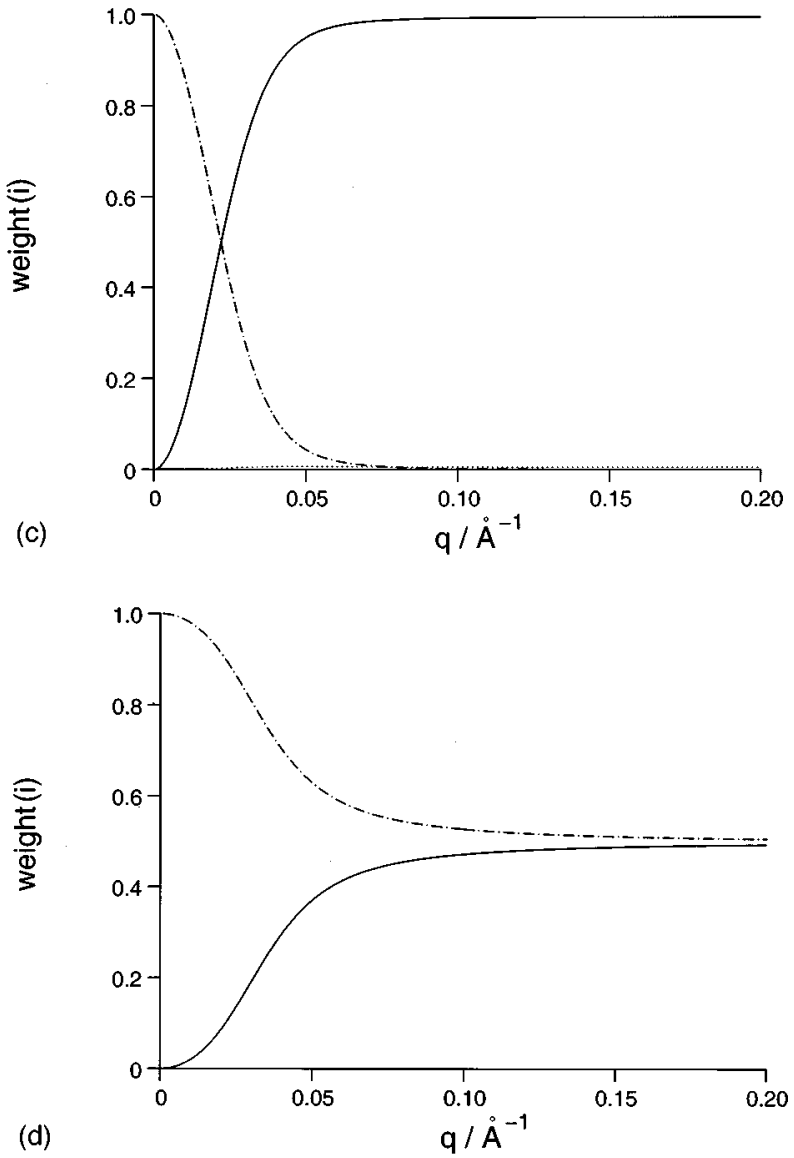

FIG. 2. (a) $Q$ dependence of the eigenvalues predicted by a four-component RPA approach for a mixture of two kinds of symmetric diblock copolymer chains differing by their labeling. $\lambda_{1}$ (solid line), $\lambda_{2}$ (dash-dotted line), $\lambda_{3}$ (dotted line). Calculations were performed with $f=0.5, R_{g \mathrm{~A}}=R_{g \mathrm{~B}}=40 \AA, N_{\mathrm{A}}=N_{\mathrm{B}}$ $=200, \kappa_{12}=0$. (b) $-(\mathrm{d})$ present the corresponding weights $\omega_{1}(q)$ (solid line) and $\omega_{2}(q)$ (dash-dotted line), $\omega_{3}(q)$ (dotted line) of the eigenvalues plotted in (a). They have been calculated for different labeling conditions: (b) mixture of $1 \%$ of $\mathrm{dA}-\mathrm{hB}$ diblock copolymer chains in a dA-dB matrix, (c) mixture of $1 \%$ of $\mathrm{hA}-\mathrm{dB}$ diblock copolymer chains in a $\mathrm{dA}-\mathrm{dB}$ matrix, (d) mixture of $1 \%$ of $\mathrm{hA}-\mathrm{hB}$ diblock copolymer chains in a $\mathrm{dA}-\mathrm{dB}$ matrix.

symmetric copolymer $\left(f=0.5, R_{g a}=R_{g b}=40 \AA, \kappa_{12}=0\right)$ as a function of $q$. While at high $q$ both eigenvalues are proportional to the momentum transfer to the fourth power (Rouse regime), at lower $q$ eigenvalue (1) becomes $q$ independent and eigenvalue (2) displays a $q^{2}$ dependence. Eigenvalue (2) has no weight and the total structure factor starts to decay with $\Gamma_{1}(q)$,

$$
I(q, t)=I(q) e^{-\Gamma_{1}(q) t} .
$$

In our example $S(q)$ would be the Leibler structure factor [Eq. (15)] and $\Gamma_{1}(q)$ describes the collective dynamics of the diblock copolymer melt. For small momentum transfers $\Gamma_{1}(q)$ may be given analytically,

$$
\begin{aligned}
\Gamma_{1}(q)= & \frac{q^{2} k_{B} T f(1-f)}{N\left[(1-f) \zeta_{a}+f \zeta_{b}\right]} \\
& \times\left[\frac{3}{2 q^{2}\left(R_{g a}^{2}+R_{g b}^{2}\right) f^{2}(1-f)^{2}}-2 \kappa_{12} N\right] .
\end{aligned}
$$

For $\kappa_{12}=0$ it describes the breathing mode of the diblock, where the two arms move with respect to a common center of mass. At $q^{*}$ for finite $\kappa_{12}, \Gamma_{1}(q)$ reaches a minimum displaying the critical slowing down of the concentration fluctuations. At $q R_{g} \gg 1$ in the Rouse limit we have

$$
\Gamma_{1}(q)=\frac{q^{4} R_{g}^{2} k_{B} T}{2 N \bar{\zeta}}\left[1-\frac{4 \kappa_{12} N}{q^{2} R_{g}^{2}} f(1-f)\right] .
$$

$\bar{\zeta}$ is an average friction coefficient which describes the collective dynamics and is given by $\bar{\zeta}=(1-f) \zeta_{a}+f \zeta_{b}$, where $N=N_{a}+N_{b}$ and $R_{g}^{2}=R_{g a}^{2}+R_{g b}^{2}$. The second mode stands for the center of mass diffusion of the diblock, which is invisible for the discussed contrast.

\section{Mixture of two different diblock copolymers}

The dynamics of a four-component polymer mixture is characterized by four different relaxation modes $\lambda_{1}, \ldots, \lambda_{4}$ which-depending on the contrast conditions-appear with weights $w_{1}(q) \cdots w_{4}(q)$ in the dynamic structure factor. These weights depend both on the volume fraction and on the contrast factors $K_{i}$ of the components in the mixture. In the following we will develop the results obtained for a mixture of two kinds of A-B diblock copolymer chains differing by their labeling. Figure 2(a) displays the $q$-dependent eigenvalues obtained for symmetric $\mathrm{A}-\mathrm{B}$ diblock copolymer chains with $N_{a}=N_{b}, f=0.5$, and $R_{g a}=R_{g b}=40 \AA$. Due to the incompressibility constraint the weight $w_{4}(q)=0$, i.e., 
the $\lambda_{4}$ mode is invisible. For our example we assume that the component $\mathrm{A}$ is the fastest with $\zeta_{b} / \zeta_{a}=4$. The eigenvalues depend on friction coefficients and the volume fraction of the components A and B and the radii of gyration of the blocks. However, they do not depend on the labeling. The observable dynamics of this system results from a weighted (weights $w_{1}, w_{2}, w_{3}, w_{4}$ ) average of these four eigenvalues representing relaxation rates.

We now discuss the nature of these relaxation modes. Thereby, we consider their appearance in the dynamic structure factor according to different chain labeling. First we relate to the case of pure $\mathrm{hA}-\mathrm{dB}$. This case is described by the eigenvalue $\lambda_{3}(q)$, which under these conditions is the only eigenvalue appearing in the dynamic structure factor $\left[w_{3}(q) \equiv 1\right] . \quad \lambda_{3}(q)$ corresponds to the collective mode $\Gamma_{1}(q)$ of the diblock copolymer melt discussed above.

As the next case, we discuss a mixture of a small fraction $\mathrm{dA}-\mathrm{hB}$ diblock chains with $\mathrm{dA}-\mathrm{dB}$ copolymer. We assume that the deuterated $\mathrm{A}$ and $\mathrm{B}$ segments have the same contrast factors. With this labeling, we will observe the motions of the protonated B monomers which are the slowest in this system. We denote the volume fraction of the $\mathrm{dA}-\mathrm{hB}$ copolymer chains as $\alpha$. Figure 2(b) shows the relative amplitudes calculated for $\alpha=0.01$.

The dynamics of such mixture is dominated over the whole $q$ range by the relaxation mode $\lambda_{2}(q)$, which thus corresponds to the dynamics of the homopolymer branch B. In the limits of small $\left(q R_{g} \ll 1\right)$ and high $\left(q R_{g} \gg 1\right)$ analytical expressions can be derived:

$$
\begin{aligned}
& \lambda_{2}(q)=\frac{q^{2} k_{B} T}{N\left((1-f) \zeta_{b}+f \zeta_{a}\right)}, \quad q R_{g} \ll 1, \\
& \lambda_{2}(q)=k_{B} T q^{4} \frac{a_{b}^{2}}{12 \zeta_{b}}, \quad q R_{g} \gg 1 .
\end{aligned}
$$

$a_{b}$ being the segment length of polymer $b$. Obviously, at low momentum transfer $\lambda_{2}$ describes the translational Rouse diffusion coefficient of the whole diblock considering $N(1$ $-f$ ) segments exerting the friction $\zeta_{b}$ and $N f$ segments exerting the friction $\zeta_{a}$. In the high $q$ limit, on the other hand, RPA predicts a strange result: The homopolymer B is predicted to undergo the same Rouse dynamics as a B homopolymer in a melt of B polymers. A proper average over the mixed surrounding is not predicted.

Now we turn to the opposite labeling and consider a small fraction of $\mathrm{hA}-\mathrm{dB}$ labeled diblocks in a $\mathrm{dA}-\mathrm{dB}$ melt. For $\alpha=1 \%$ the corresponding relative weight factors of the eigenvalues are displayed in Fig. 2(c). Now at high $q$ basically all weight is with eigenvalue $\left(\lambda_{1}\right)$, which we interpret as the motion of the A arm in the diblock copolymer melt. At lower $q$ in the region where the static structure factor under hA-dB labeling shows its maximum $\left(q^{*}\right)$ the weight crosses over to mode $\left(\lambda_{2}\right)$, the mode describing the translational diffusion of the whole molecule. Thus, no matter which arm we label at low $q$ always the translational diffusion of the total diblock is seen, as it should be. Again also for mode $\lambda_{1}(q)$ we give the asymptotic values for small and large $q$,

$$
\begin{aligned}
& \lambda_{1}(q)=\frac{3}{2} \frac{\left[(1-f) \zeta_{b}+f \zeta_{a}\right] k_{B} T}{N \zeta_{a} \zeta_{b}\left(R_{g a}^{2}+R_{g b}^{2}\right) f(1-f)}, \quad\left(q R_{g}\right) \ll 1, \\
& \lambda_{1}(q)=\frac{k_{B} T q^{4} a_{a}^{2}}{12 \zeta_{a}}, \quad\left(q R_{g}\right) \gg 1 .
\end{aligned}
$$

Again at high $q$ the RPA predicts that the dynamics of arm A is identical to the Rouse motion of an A polymer in an A-homopolymer melt. At low $q, \lambda_{1}(q)$ turns into a breathing mode with a nonvanishing relaxation rate at $q=0$, as the collective mode $\lambda_{3}(q)$.

Finally, we consider a mixture of an $\mathrm{hA}-\mathrm{hB}$ diblock in a deuterated matrix dA-dB. Figure 2(d) presents the corresponding $q$-dependent relative weights for $\alpha=1 \%$. Under these conditions at high $q$ the theory predicts the observation of the weighted average of two different homopolymerlike Rouse rates, while at low $q$ again the weight turns toward the diffusive mode $\left(\lambda_{2}\right)$. The then breathing mode $\lambda_{1}$ approaches the weight zero.

Finally, we would like to note: While the eigenvalues of $\Omega$ describe relaxation properties inherent to the polymer system under consideration, the initial slopes discussed at the end of Sec. II C [Eqs. (32) and (33)] depend on the observation conditions and thus on the chosen labeling. In general the eigenvalues combined with the weights may be transferred directly into initial slopes which are meaningful from an experimental point of view.

Caution is advisable-as now is exemplified-for the case of labeling a small fraction of the A blocks in the system. Looking at the weights displayed in Fig. 2(c) we realize that at low $q$ weight crosses over from eigenvalue $\lambda_{1}$ to eigenvalue $\lambda_{2}$. At the same time the values of $\lambda_{1}$ and $\lambda_{2}$ become grossly different. In an actual experiment clearly a relaxation corresponding to $\lambda_{2}$ would be observed. However, calculating the initial slope following Eq. (33) leads to

$$
\Gamma(q)=\frac{k T q^{2}}{N_{a} \zeta_{a}} .
$$

Thus, the initial slope corresponds to that of an A arm performing translational diffusion without knowing about the slower arm B. Similarly also the prediction for a small fraction of labeled B arms leads to the unphysical result $\Gamma(q)$ $=k T q^{2} / N_{b} \zeta_{b}$, again a single arm performing center of mass diffusion.

Applying the Gaussian approximation, the dynamic structure factor $S(q, t)$ which is measured in neutron spin echo experiments can be written in terms of mean square segment displacements $\left\langle\left(r_{n}(t)-r_{m}(0)\right)^{2}\right\rangle$ as

$$
S(q, t)=\frac{1}{N} \sum_{n} \sum_{m} \exp \left\{-\frac{q^{2}}{6}\left\langle\left(r_{n}(t)-r_{m}(0)\right)^{2}\right\rangle\right\} .
$$

For $q R_{g} \gg 1$ and times to $t \ll \tau_{R}$ DeGennes ${ }^{26}$ has derived an analytic form which-neglecting translational diffusionmay be expressed as an integral,

$$
S(q, t)=\frac{12}{q^{2} l^{2}} \int_{0}^{\infty} d y \exp \left[-y-\left(\Gamma_{q} t\right)^{1 / 2} k\left(y\left(\Gamma_{q} t\right)^{-1 / 2}\right)\right]
$$


with

$$
k(y)=\frac{2}{\pi} \int_{0}^{\infty} d x \frac{\cos (x y)}{x^{2}}\left(1-e^{-x^{2}}\right), \quad \Gamma_{q}=\frac{k T}{12 \zeta} q^{4} l^{4} .
$$

In this expression $S(q, t)$ depends on one single scaling variable $u=q^{2} \sqrt{W l^{4} t}$. $W l^{4}$ is called the Rouse rate and is given by $W l^{4}=3 \mathrm{kTl}^{2} / \zeta$ with $\zeta$ the friction coefficient of a segment of length $l$.

The first cumulant $\Gamma_{R}$, of Eq. (40) becomes:

$$
\Gamma_{R}=\frac{1}{36}\left(\frac{3 k T l^{2}}{\zeta}\right) q^{4} \text {. }
$$

Accounting also for translational diffusion Eq. (41) transforms into

$$
\Gamma_{R}=\frac{W l^{4}}{36} q^{2}\left(q^{2}+\frac{2}{R_{g}^{2}}\right) .
$$

\section{EXPERIMENT}

\section{A. Samples}

Three different polymer systems were investigated: PEE homopolymer and diblocks of PE-PEE and PEP-PEE. All polymers were synthesized by anionic polymerization of butadiene and isoprene monomers followed by subsequent hydrogenation or deuteration. ${ }^{27}$ Sample I was a blend of fully protonated and fully deuterated polyethylethylene (hPEE/dPEE) with the same degrees of polymerization. These polymers were derived from linear 1,2-polybutadiene chains. Short symmetric diblock copolymers of polyethylene-block-polyethylethylene (PE-PEE) were obtained by deuteration of 1,4-polybutadiene-block-1,2-polybutadiene copolymers, thus leading to a nominal composition of $h_{6} d_{2}$ of the protonated repeat unit. Samples II and III were blends of the deuterated dPE-dPEE matrix with $20 \%$ of partially protonated hPE-dPEE and dPE-hPEE, respectively. Sample IV was the pure partially protonated hPE-dPEE diblock.

Samples V and VI contained long copolymer chains of poly(ethylene-propylene)-block-polyethylethylene(PEPPEE). The parent materials of these block copolymers were 1,4-polyisoprene-block-1,2-polybutadienes. Sample V was a deuterated matrix dPEP-dPEE containing $10 \%$ of protonated hPEP-hPEE while sample VI was pure partially protonated dPEP-hPEE (nominal $d_{2} h_{6}$ composition of PEE repeat unit) block copolymers. The use of mixtures with partially deuterated components (samples I-III, and V) allowed us to analyze single chain dynamics while collective chain dynamics is pooled on samples IV and VI. Table I summarizes all samples and their characterization.

\section{B. Small angle neutron scattering (SANS)}

The SANS experiments were performed at the KWS1 instrument at the research reactor FRJ2 of the Forschungszentrum Jülich and at D17 at the Institut Laue Langevin (ILL) in Grenoble, France. In Jülich we studied sample IV (hPE-dPEE), which was put into $1 \mathrm{~mm}$ copper cell with quartz windows. The cell was filled under inert Ar atmo-

\begin{tabular}{|c|c|c|c|c|}
\hline Polymer & $\begin{array}{c}M_{w} \\
(\mathrm{~g} / \mathrm{mol})\end{array}$ & $M_{w} / M_{n}$ & $\begin{array}{l}N_{\mathrm{PEE}} \\
\text { (PEE monomer } \\
\text { number) }\end{array}$ & $\begin{array}{c}f_{\mathrm{PEE}} \\
\text { (volume fraction } \\
\text { in PEE) }\end{array}$ \\
\hline hPEE & 21550 & 1.02 & 385 & $\ldots$ \\
\hline dPEE & 24530 & 1.02 & 383 & $\cdots$ \\
\hline \multicolumn{5}{|c|}{ Sample I is a mixture of $20 \%$ hPEE in dPEE } \\
\hline hPE-dPEE & 16900 & 1.02 & 127 & 0.5 \\
\hline dPE-hPEE & 16500 & 1.02 & 136 & 0.5 \\
\hline dPE-dPEE & 16400 & 1.02 & 130 & 0.5 \\
\hline \multicolumn{5}{|c|}{$\begin{array}{l}\text { Sample II: } 20 \% \text { hPE-dPEE in dPE-dPEE } \\
\text { Sample III: } 20 \% \text { dPE-hPEE in dPE-dPEE } \\
\text { Sample IV: hPE-dPEE }\end{array}$} \\
\hline hPEP-hPEE & 68000 & 1.03 & 553 & 0.45 \\
\hline dPEP-hPEE & 68000 & 1.03 & 533 & 0.45 \\
\hline dPEP-dPEE & 68000 & 1.03 & 480 & 0.45 \\
\hline \multicolumn{5}{|c|}{$\begin{array}{l}\text { Sample V: } 10 \% \text { of hPEP-hPEE in dPEP-dPEE } \\
\text { Sample VI: hPEP-dPEE }\end{array}$} \\
\hline
\end{tabular}

TABLE I. Compilation of samples used in the experiments described in the text.

sphere in order to avoid the degradation of the polymer chains at high temperature. The experiments covered a temperature range $373 \mathrm{~K} \leqslant T \leqslant 473 \mathrm{~K}$ and $q$ range 0.01 $\leqslant 0.1 \AA^{-1}$. Altogether seven temperatures were studied. The obtained data were corrected for background and detector sensitivity.

Similarly, sample VI (dPEP-hPEE) was investigated at D17 at the ILL. There the temperature region around the order-disorder transition temperature $T_{\mathrm{ODT}}=473 \mathrm{~K}$ was covered $373 \mathrm{~K} \leqslant T \leqslant 533 \mathrm{~K}$ studying a $q$ range $0.01 \leqslant q$ $\leqslant 0.12 \AA^{-1}$ at four different temperatures. Again the data underwent standard correction procedures and were converted to absolute cross sections.

\section{Neutron spin-echo experiments (NSE)}

The high resolution inelastic scattering has been performed using the neutron spin-echo (NSE) spectrometer at the Forschungszentrum Jülich for most of the samples and the NSE spectrometer IN11 at the ILL, Grenoble, France for the 10\% hPEP-hPEE in the dPEP-dPEE sample. The samples were filled in rectangular niobium cuvettes under argon atmosphere. The sample thickness was $2 \mathrm{~mm}$ for samples IV and VI and $4 \mathrm{~mm}$ for samples I-III and V. The illuminated sample area was $30 \times 30 \mathrm{~mm}^{2}$. To account for the background scattering (mainly deeply inelastic multiple scattering, residual SANS from the cuvette windows, etc.) background samples of dPEE, dPE-dPEE, and dPEP-dPEE were investigated and subtracted with the proper correction factors. Measurements were performed at two temperatures473 and $533 \mathrm{~K}$. For the NSE spectrometer in Jülich, ${ }^{28}$ the momentum transfer covered was $0.03 \AA^{-1}<q<0.22 \AA^{-1}$ using five different detector arm settings ( $q=0.05,0.08,0.1$, $0.14,0.20 \AA^{-1}$ ), where each setting allowed for the extraction of three to four different adjacent $q$ values within the multidetector range of $\pm 0.027 \AA^{-1}$ per setting. We achieved a $q$-resolution 


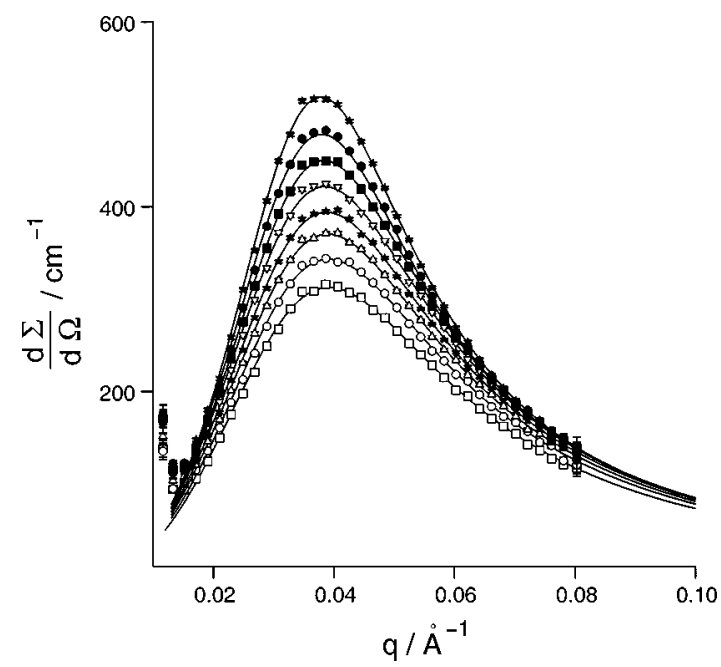

FIG. 3. Variation of the static structure factor $\mathrm{S}(\mathrm{q})$ measured on the hPEdPEE diblock copolymer chains (sample IV) as a function of the wave number $q$. Temperature: (closed star): $393 \mathrm{~K}$, (closed circles): $403 \mathrm{~K}$, (closed square): $413 \mathrm{~K}$, (inverted triangle): $423 \mathrm{~K}$, (closed star): $433 \mathrm{~K}$, (open triangle): $443 \mathrm{~K}$, (open circle): $453 \mathrm{~K}$, (open square): $463 \mathrm{~K}$. Solid lines represent the fit with a two-component static RPA approach [Eq. (12)].

$$
\Delta q_{\mathrm{FWHM}}=\sqrt{\left(0.01 \AA^{-1}\right)^{2}+\left(q \times \frac{\Delta \lambda}{\lambda}\right)^{2}},
$$

i.e., $0.01 \AA^{-1}<\Delta q_{\text {FWHM }}<0.02 \AA^{-1}$ using a wavelength $\lambda$ $=8 \AA$ with a spread $(\Delta \lambda / \lambda)_{\mathrm{FWHM}}=0.1$. The time range was $0.1<t<22 \mathrm{~ns}$ covered by 14 settings. The measuring time was 1 day per sample and temperature. The reduction of the multidetector data to the $S(q, t) / S(q)$ curves followed the procedures described in Ref. 28.

Measurements performed on IN11 at ILL were made at a wavelength $\lambda=8.1 \AA$ allowing us to achieve times up to 20 ns. The accessible $q$ range amounted to $0.02 \AA^{-1}<q$ $<0.165 \AA^{-1}$ with a $q$ resolution of about $20 \%$.

\section{RESULTS}

\section{A. SANS measurements}

The morphology of a diblock copolymer is controlled by the competition between the enthalpic and entropic contributions to the free energy. The enthalpy stems from the repulsion between segments of different chemical nature. The degree of incompatibility is characterized by the Flory Huggins interaction parameter $\kappa_{12}$ [see Eq. (7)]. Generally at high temperature the entropic contribution is dominant and the system appears to be uniformly mixed showing weak spatial inhomogeneities or concentration fluctuations. At low temperature the enthalpy wins and the system tries to minimize the number of unfavorable contacts between chemically different segments. Therefore, it undergoes a microphase separation characterized by long range order in its composition. The morphology of a diblock copolymer is directly reflected by the static structure factor measured by SANS.

Figures 3 and 4 present the temperature-dependent static structure factors measured on the hPE-dPEE (sample IV) and dPEP-hPEE (sample VI) copolymer chains, respectively. At intermediate $q$ the typical peak structure for a diblock co-

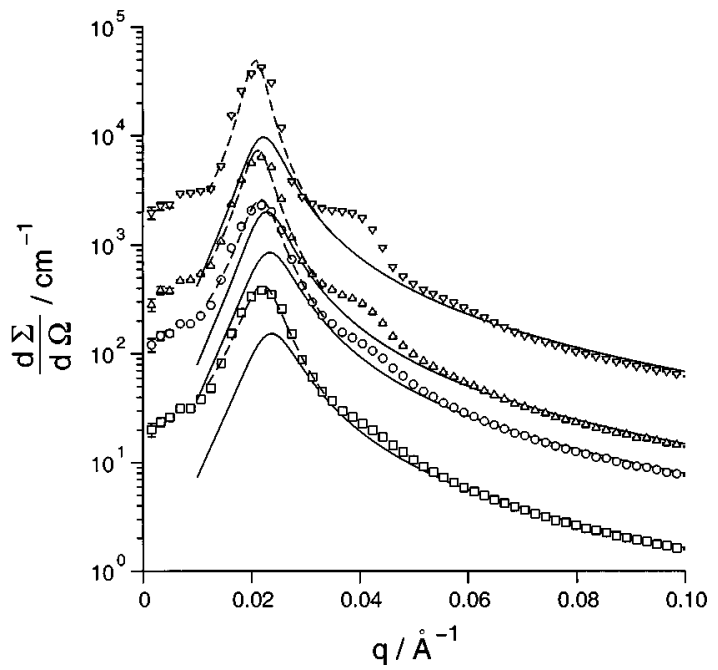

FIG. 4. $Q$ dependence of the logarithm of the static structure factor $S(q)$ measured on the hPEP-dPEE diblock copolymer chains (sample VI). Temperature: (square): $533 \mathrm{~K}$, (circle): $518 \mathrm{~K}$, (triangle): $498 \mathrm{~K}$, (inverted triangle): $478 \mathrm{~K}$. The dashed lines represent the fit performed with a Lorentzian on the $q$ range $0.01 \AA^{1}<q<0.035 \AA^{-1}$ in order to determine the width and the position of the peak. The solid lines represent the fit using a twocomponent static RPA approach [Eq. (12) and (43)] for $0.01 \AA^{-1}<q$ $<0.035 \AA^{-1}$. For a better understanding, the fit and experimental data have been shifted using a factor $k: 533 k: k=50,518 \mathrm{~K}: k=10,498 \mathrm{~K}: k=5$, $478 \mathrm{~K}: k=1$.

polymer is observed which results from the block structure of the molecule. For the PE-PEE system a peak position near $q^{*} \cong 0.036 \AA^{-1}$ is found while for PEP-PEE as a consequence of the higher molecular weight $q^{*} \cong 0.02 \AA^{-1}$ is observed.

For the PE-PEE system, at temperatures below $373 \mathrm{~K}$ crystallization occurs. This diblock copolymer does not undergo the order-disorder microphase separation: the peak stays broad above $373 \mathrm{~K}$, where the system is in the mean field regime and concentration fluctuations are low. The data were analyzed in terms of the RPA structure factor [Eq. (12)] where we used expressions for the partial structure factors allowing for the different segment lengths and thus different radii of gyration of the two blocks: $:^{15}$

$S_{i i}^{0}=\phi_{i} N_{i} P_{i i}\left(N_{i}, \alpha_{i}\right)$,

$S_{j i}^{0}=\sqrt{\phi_{i} N_{i} \phi_{j} N_{j}} P_{i j}\left(N_{i}, N_{j}, \alpha_{i}, \alpha_{j}\right)$,

$P_{i i}\left(N_{i}, a_{i}\right)=\frac{1}{N_{i}}\left\{1+2\left(e^{a_{a}}-1\right)^{-1}\left[1-\frac{\left(1-e^{-N_{i} a_{i}}\right)}{N_{a}\left(1-e^{-a_{a}}\right)}\right]\right\}$

$P_{i j}\left(N_{i}, N_{j}, \alpha_{i}, \alpha_{j}\right)$

$$
=\frac{1}{N_{i} N_{j}} e^{-\left(\alpha_{i}+\alpha_{j}\right) / 2}\left[\frac{\left(1-e^{-\alpha_{i} N_{i}}\right)\left(1-e^{-\alpha_{j} N_{j}}\right)}{\left(1-e^{-\alpha_{i}}\right)\left(1-e^{-\alpha_{j}}\right)}\right],
$$

$\alpha_{i}=\frac{q^{2} a_{i}^{2}}{6}$.

Thereby $a_{i}$ is the segment length of an $i$ chain, $N_{i}$ is the number of segments, and $\phi_{i}$ is the volume fraction of the monomers of type " $i$." In order to keep the number of parameters small and keeping in mind that this sample was far from the ODT, we fixed the ratio of the $a_{i}$ according to the 


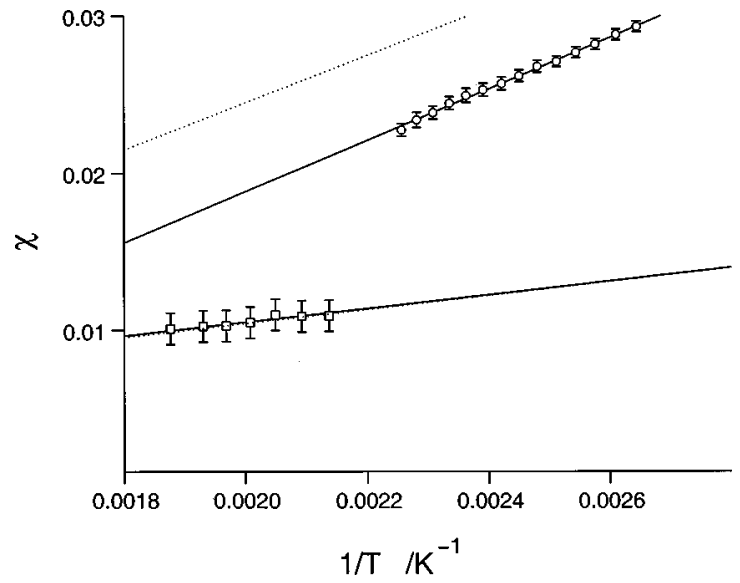

FIG. 5. Variation of the Flory-Huggins interaction parameter vs the inverse of the temperature (1/T): hPE-dPEE diblock (circle), dPEP-hPEE: (square). Our experimental data are compared with the results obtained by Bates et al. for both systems [dotted lines: up (PE-PEE); down (PEP-PEE)].

ratio of the radii of gyration of $\mathrm{PE}$ and $\mathrm{PEE}$ to what is known from the literature $R_{g}^{\mathrm{PEE}}=R_{g}^{0 \mathrm{PEE}} \exp \left(1 \times 10^{-4} K^{-1}\left(T-T_{0}\right)\right)^{29}$ and $R_{g}^{\mathrm{PE}}=R_{g}^{0 \mathrm{PE}} \exp \left(-7 \times 10^{-4} K^{-1}\left(T-T_{0}\right)\right) .{ }^{29}$ With the total radius of gyration $R_{g}$ in terms of $a_{i}$, the $\kappa_{\mathrm{PE}-\mathrm{PEE}}$ parameter and the total intensity as fitting variables the data were fitted with Eq. (12) using Eq. (43). As can be seen from the solid lines in Fig. 3 a very good data description is achieved. Figure 6 presents the resulting radius of gyration $R_{g}(T)$ which can be described by an exponential temperature dependence with an expansion coefficient $H_{\mathrm{PE}-\mathrm{PEE}}=-5$ $\times 10^{-4} / \mathrm{K}$, a value close to the average between PEE and PE. The absolute value of $R_{g}$, however, comes out to about $10 \%$ larger than what would be expected from the radii of the single arms [e.g., $473 \mathrm{~K}: R_{g}=49 \AA ; \sqrt{\left(R_{g}^{\mathrm{PE}}\right)^{2}+\left(R_{g}^{\mathrm{PEE}}\right)^{2}}$ $=44 \AA$ ] . Considering recent results from Rosedale et al., ${ }^{30}$ who found that chain stretching sets in around $\kappa N \geqslant 6$, our observation of chain stretching underpins this earlier finding (for our system we have $6<\kappa N<7$ ). Figure 5 displays the obtained temperature dependent Flory-Huggins parameter $\kappa_{\mathrm{PE}-\mathrm{PEE}}(T)$ as a function of $1 / T$. We find the relation $\kappa_{\mathrm{PE}-\mathrm{PEE}}=14.5 \mathrm{~K} / T-0.0106$. For comparison we also show the earlier results of Rosedale et al. by a dashed line. We observe the same temperature dependence but a slightly different entropic contribution.

Other than for the previous polymer system, SANS measurements performed on dPEP-hPEE chains show the presence of an order-disorder transition which occurs at $473 \mathrm{~K}$. The peak structure is much more pronounced and secondorder maxima are observed, which cannot be described in the RPA formalism (Fig. 3). In order to obtain precise data for the radius of gyration $R_{g}$ and on the Flory-Huggins parameter in a first attempt, we selected the peak region of the first peak only $\left(0.01 \AA^{-1}<q<0.035 \AA^{-1}\right)$ and fitted these data with a Lorentzian giving the position of the peak $q^{*}$ and its width $\Gamma$. From the peak position we derive the radius of gyration $\left(q^{2} R_{g}^{2}=3.7995\right.$ according to Fredrickson $\left.{ }^{10}\right)$. The effective Flory-Huggins parameter $\kappa_{12}^{\text {eff }}$ is determined from the correlation length and the radius of gyration of the chains according to ${ }^{10}$

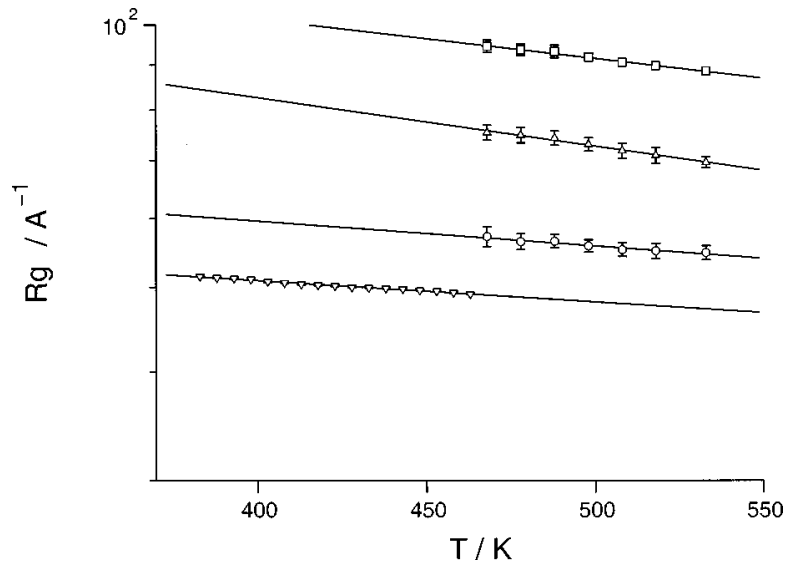

FIG. 6. Temperature dependence of the logarithm of the radii of gyration of the hPE-dPEE chains (inverted triangle) and the dPEP-hPEE chains (square), of the PEE block (circles) and the PEP block (triangle) of the dPEP-hPEE diblock copolymer chains.

$$
\Gamma=\sqrt{\frac{4 x^{*} F^{\prime \prime}\left(x^{*}\right) R_{g}^{2}}{2\left(F^{*}-2 \kappa_{12}^{\mathrm{eff}} N\right)}},
$$

where $x^{*}=3.7995, F^{\prime \prime}\left(x^{*}\right)=0.9688$, and $F^{*}=21.396 . R_{g}$ and $N$ are the radius of gyration and the number of segment of the diblock copolymer chain, respectively. The FloryHuggins parameter $\kappa_{\text {PEP-PEE }}$ then follows from Eq. (44).

The resulting temperature-dependent values for $R_{g}$ and $\kappa_{\text {PEP-PEE }}$ are displayed in Figs. 5 and 6 . Other than for the short chain PE-PEE molecule for PEP-PEE we observe a strong temperature variation of $R_{g}=94.3 \exp [-1.0$ $\left.\times 10^{-3} K^{-1}\left(T-T_{0}\right)\right][\AA]\left(T_{0}=473 \mathrm{~K}\right)$ considerably stronger than what would be expected from the chain expansion of the homopolymers. Here stretching effects in the neighborhood of $T_{\mathrm{ODT}}$ contribute significantly (see also Bates et $\left.a l .{ }^{27}\right)$. The Flory-Huggins parameter displays a linear regression in $1 / T$ following the relation $\kappa_{\mathrm{PEP}-\mathrm{PEE}}^{\text {eff }}=4.27 \mathrm{~K} / T$ $+1.8 \times 10^{-3}$. In order to compare with earlier SANS-based data we include the results of Rosedale et al. ${ }^{30}$ by a dashed line in Fig. 6. The agreement is remarkable. Note that exactly these $\kappa$ parameters that describe $S(Q)$ well within the RPA are used as input for the dynamical RPA the results of which are compared to experiments on the same samples.

Finally, in order to compare with the RPA structure factor, we also fitted Eq. (12) together with Eq. (43) to the data. Thereby, particular emphasis was placed on the flanks of the structure factor of the $q$ range, where the NSE experiments were performed. These fits used $\kappa_{12}$ and $R_{g}$ from the Lorentz description as constraints and varied the radii of gyration of the two components separately. The solid lines in Fig. 4 represent the results which agree well in the flanks but show deficiencies in the description of the main peak. The following results are obtained: $R_{g}^{\mathrm{PEP}}=75 \exp \left[-1.2 \times 10^{-4} K^{-1}(T\right.$ $\left.\left.-T_{0}\right)\right][\AA] \quad$ and $R_{g}^{\mathrm{PEE}}=56.9 \exp \left[-7 \times 10^{-4} K^{-1}\left(T-T_{0}\right)\right]$ $\left(T_{0}=473 \mathrm{~K}\right)$ (see also Fig. 5). Considering the absolute values of $R_{g}$, we observe that the two blocks are stretched by $10 \%$ in comparison with the corresponding PEE and PEP homopolymers. ${ }^{31,29}$ 


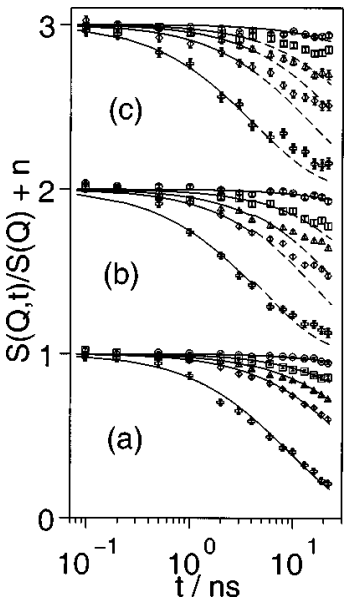

FIG. 7. NSE spectra from: (a) the PEE melt (sample I), (b) the $20 \%$ labeled hPE-dPEE fraction in a deuterated PE-PEE matrix (sample II), (c) the hPEdPEE diblock copolymer melt (sample IV). $Q$ values in each case from above, $Q / \AA^{-1}=0.05,0.08,0.10,0.121,0.187$. The solid lines are the result of a fit with a Rouse dynamic structure factor. Their extent marks the fitting range; the dashed lines extrapolate the Rouse structure factor. All data were taken at $473 \mathrm{~K}$

\section{B. Dynamics}

The theoretical considerations developed in Sec. II are concerned with the short time relaxation behavior or the initial slope of the relaxation function. Deviations from the initial relaxation could in principle be treated by introducing memory terms into the equations of motion [see Eq. (19)]; an approach that leads to considerable mathematical complications. For our data evaluation we chose an approximation which accounts for the actual line shape of the Rouse relaxation function [Eq. (40)] neglecting any further effects due to, e.g., entanglements or intrinsic line shapes induced by possibly complex relaxation processes in the diblock melt. This approach allows a stable fit over a time regime large enough to obtain accurate results. The fitted values for the Rouse factor yield the initial slopes required by the RPA theory [see, e.g., Eq. (41)].

\section{Results on the PEE homopolymer}

Neutron spin-echo measurements were performed on a mixture of $20 \%$ of hPEE in dPEE at two temperatures - 473 and $533 \mathrm{~K}$. Figure 7 (a) shows the time dependence of the intermediate dynamic structure factor measured at $473 \mathrm{~K}$ at different $q$ values. These experimental data were fitted with the Rouse dynamic structure factor [Eq. (40)]. The only free parameter is the Rouse factor $W l^{4}=3 k T l^{2} / \zeta$, which does not depend on $q$.

As can be seen from Fig. 7(a), we obtain good agreement between the Rouse prediction and the data over the full time and $q$ range. Furthermore, in order to test for the $q$ dependence of the spectra we also fitted the experimental data separately for each $q$ value. By this procedure we become sensitive with respect to possible variations of the Rouse factor with $q$, while the joint fit mentioned above reveals the average value $\left\langle W l^{4}\right\rangle=0.8 \times 10^{13} \AA^{4} \mathrm{~s}^{-1}$ for the Rouse factor. The maximal deviation of the $q$-dependent Rouse factor from the average $\left\langle W l^{4}\right\rangle$ amounts to 0.1 $\times 10^{13} \AA^{4} \mathrm{~s}^{-1}$.

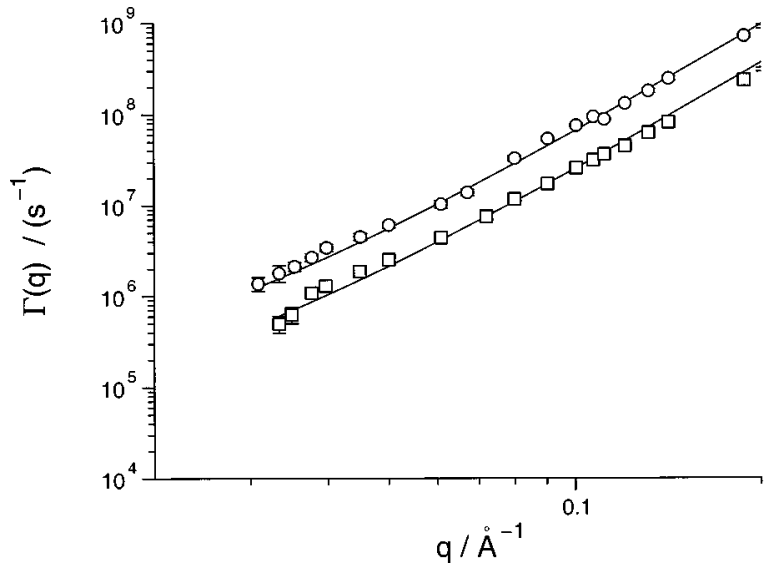

FIG. 8. Rouse relaxation rates from the PEE melt at (square) $473 \mathrm{~K}$ and (circle) $533 \mathrm{~K}$ with the prediction of the Rouse model (solid line) given by Eq. (42).

The same procedure was applied at $533 \mathrm{~K}$ with the result of $\left\langle W l^{4}\right\rangle=2.2 \times 10^{13} \AA^{4} \mathrm{~s}^{-1}$. The $q$ dependence of the first cumulant $\Gamma_{R}$ [see Eq. (42)] for the PEE at the two temperatures is plotted in Fig. 8. The solid lines display the prediction of Eq. (42) taking for the Rouse factor the average $\left\langle W l^{4}\right\rangle$. The filled squares and rings correspond to the results of the individual fits performed at each $q$ value at 473 and $533 \mathrm{~K}$, respectively. We observe an excellent agreement with the theoretical expectation. Finally, Table II summarizes the results at both temperatures.

\section{The dynamics of single chains in the block copolymer melt}

As outlined in Sec. II D labeling of a small fraction of components of one kind allows direct access to the single chain dynamics of this component. Such experiments were carried out on samples II and III, where we studied the dynamics of the PE or the PEE component within the short chain PE-PEE diblock. Furthermore, the structure factor of a fully labeled PEP-PEE chain within a PEP-PEE matrix was investigated. Considering the $q$ values accessible to NSE measurements, we analyze in the case of short PE-PEE chains the dynamics at length scales both smaller and larger than the structural correlation domains while we only probe the dynamics for the fluctuations smaller than the structural correlation domains with the long chain PEP-PEE.

We first present the results measured on the PE-PEE system. Figures 7(b) and 7(c) show the variation of $S(q, t) / S(q)$ measured for sample II $(20 \%$ hPE-dPEE in dPE-dPEE) and sample III (20\% dPE-hPEE in dPE-dPEE), respectively. The Rouse factors were determined following the same procedure as for the PEE homopolymer. Other than for pure PEE, however, we had to restrict the fitting range in time $(t<6 \mathrm{~ns})$, because at longer times deviations from the Rouse dynamics due to entanglement constraints become visible. ${ }^{30}$ These effects are not studied here.

As has been outlined before, a description of the above experimental situation within the RPA frame needs a fourcomponent approach, the mixture of two different (by labeling) diblock copolymers (each bringing in two components) 
TABLE II. Experimental Rouse rates for the pure homopolymers and for the block-copolymer melts obtained from the high $q$ behavior of $\Gamma(q)$ compared to the predictions of the RPA theory. Values of $W l^{4}$ are given in $10^{-13} \AA^{4} \mathrm{~s}^{-1}$.

\begin{tabular}{|c|c|c|c|c|c|c|c|c|}
\hline \multirow{2}{*}{$\begin{array}{c}T \\
(\mathrm{~K})\end{array}$} & \multirow{2}{*}{$\begin{array}{l}W l^{4} \\
\mathrm{PEE}\end{array}$} & \multirow{2}{*}{$\begin{array}{c}W l^{4} \\
\text { PE } \\
\text { Ref. } 15\end{array}$} & \multicolumn{2}{|c|}{$\begin{array}{c}W l^{4} \\
20 \% \text { hPE-dPEE } \\
\text { in dPE-dPEE }\end{array}$} & \multicolumn{2}{|c|}{$\begin{array}{c}W l^{4} \\
\text { hPE-dPEE }\end{array}$} & \multicolumn{2}{|c|}{$\begin{array}{c}W l^{4} \\
20 \% \text { dPE-hPEE } \\
\text { in dPE-dPEE }\end{array}$} \\
\hline & & & (a) & (b) & (a) & (b) & (a) & (b) \\
\hline 473 & $\begin{aligned} & 0.8 \\
\pm & 0.15\end{aligned}$ & $\begin{aligned} & 4.2 \\
\pm & 0.15\end{aligned}$ & $\begin{array}{c}2 \\
\pm 0.2\end{array}$ & 3.8 & $\begin{aligned} & 1.8 \\
\pm & 0.15\end{aligned}$ & 1.7 & $\begin{array}{r}0.97 \\
\pm 0.15\end{array}$ & 0.87 \\
\hline 533 & $\begin{array}{r}2.2 \\
\pm 0.2\end{array}$ & $\begin{array}{c}8 \\
\pm 0.1\end{array}$ & $\begin{array}{r}4.1 \\
\pm 0.4\end{array}$ & 7.4 & $\begin{array}{r}3.5 \\
\pm 0.2\end{array}$ & 3.6 & $\begin{array}{r}2.5 \\
\pm 0.2\end{array}$ & 2.3 \\
\hline
\end{tabular}

$W l^{4}$

\begin{tabular}{|c|c|c|c|c|c|c|}
\hline \multirow{2}{*}{$\begin{array}{c}T \\
(\mathrm{~K})\end{array}$} & \multirow{2}{*}{$\begin{array}{l}W l^{4} \\
\mathrm{PEE}\end{array}$} & \multirow{2}{*}{$\begin{array}{c}W l^{4} \\
\text { PEP } \\
\text { Ref. } 14\end{array}$} & \multicolumn{2}{|c|}{$\begin{array}{l}\text { 10\% hPEP-hPEE } \\
\text { in dPEP-dPEE }\end{array}$} & \multicolumn{2}{|c|}{$\begin{array}{c}W l^{4} \\
\text { hPEP-dPEE }\end{array}$} \\
\hline & & & (a) & (b) & (a) & (b) \\
\hline 473 & 0.8 & 3 & $1.35 \pm$ & 1.8 & 1.5 & 1.4 \\
\hline & \pm 0.15 & \pm 0.1 & 0.2 & & \pm 0.15 & \\
\hline 533 & $\begin{array}{r}2.2 \\
+02\end{array}$ & $\begin{array}{r}4.4 \\
+02\end{array}$ & $\begin{array}{r}3.4 \\
+\quad 03\end{array}$ & 3.6 & $\begin{array}{c}3 \\
+02\end{array}$ & 3.2 \\
\hline
\end{tabular}

${ }^{a}$ Experimental values.

${ }^{\mathrm{b}}$ Rouse factor given by the RPA theory.

within a fictive homopolymer matrix " 0 " the volume fraction of which is considered to disappear. In this calculation the bare mobilities are taken from the corresponding homopolymers, the structure factor data relate to the static experiments - thus there is no free parameter left.

Figure 9 compares the experimental results with the thus
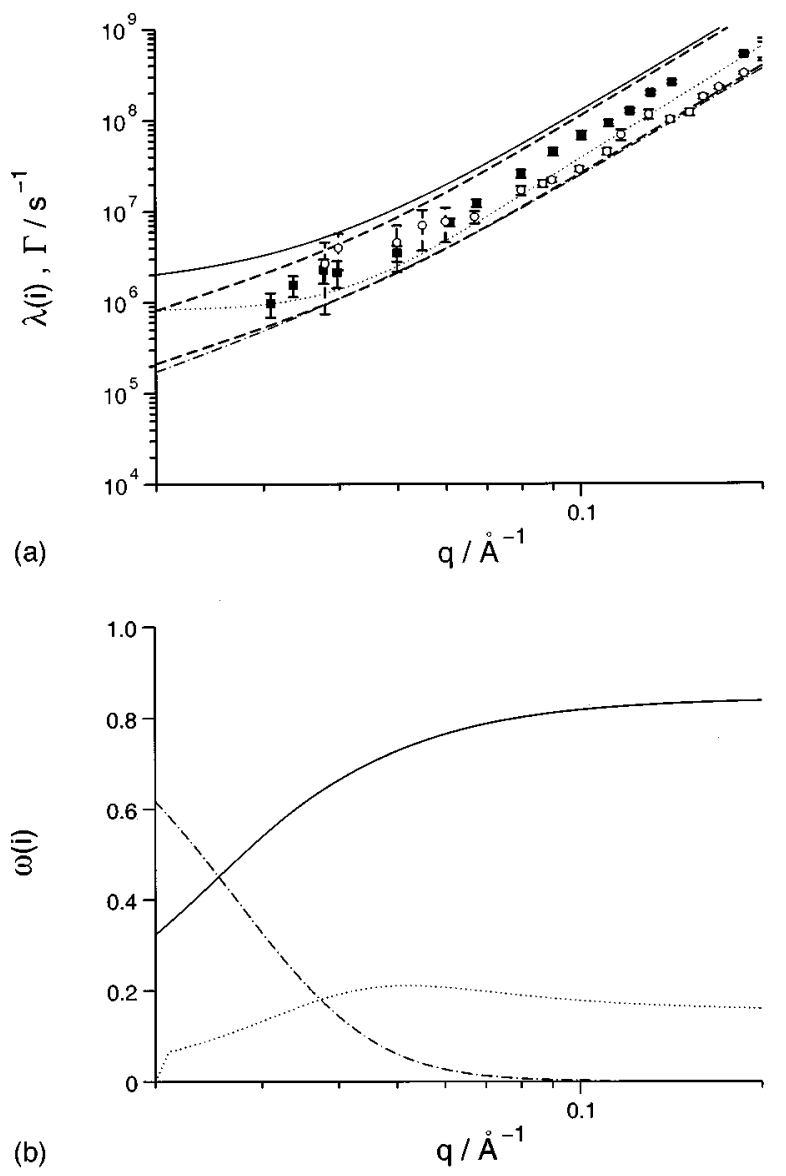

obtained RPA predictions from Sec. IID. Figure 9(a) displays the $q$-dependent eigenvalues as well as RPA initial slopes together with the experimental initial slopes obtained from samples II (PE label) and sample III (PEE label). Fig-

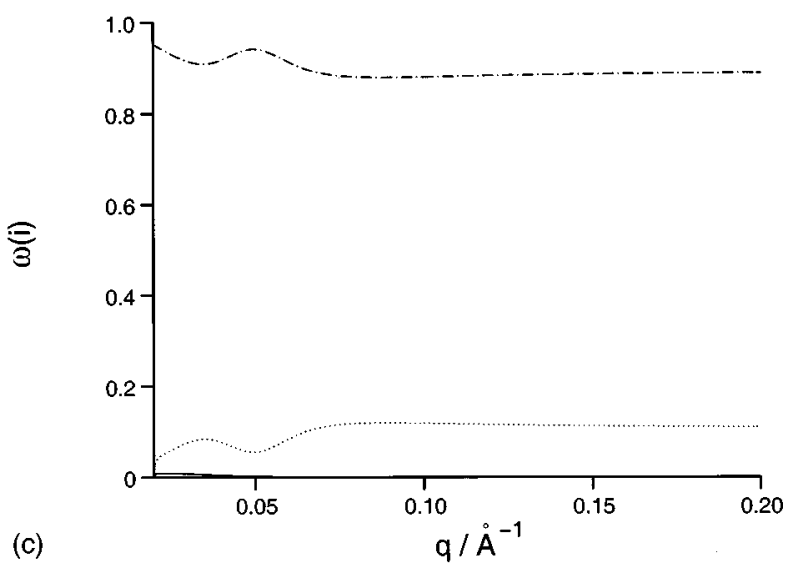

FIG. 9. (a) Rouse relaxation rates from the labeled PE arm (closed square) and the labeled PEE arm (open circle) of a PE-PEE diblock copolymer in a deuterated diblock copolymer matrix at $473 \mathrm{~K}$. The various lines represent eigenvalues derived from the four-component dynamic RPA approach: $\lambda_{1}$ (solid line), $\lambda_{2}$ (dash-dotted line), $\lambda_{3}$ (dotted line). (b) and (c) The relative weights $\omega_{1}$ (solid line), $\omega_{2}$ (dash-dotted line), $\omega_{3}$ (dotted line) of the eigenvalues plotted in (a) for several labeling conditions. (b) Relative weights for a mixture of a $20 \%$ of hPE-dPEE diblock chains in a dPE-dPEE matrix (sample II). (c) Relative weights for a mixture of a 20\% of dPE-hPEE diblock chains in a dPE-dPEE matrix (sample III). The first cumulant which is the weighted average of the eigenvalues is represented in dashed line (a): upper dashed line: first cumulant of sample II; lower dashed line: first cumulant of sample III. 
ures 9(b) and 9(c) show the weights of the different modes in the dynamic structure factor which are determined by the chain labeling. While in the case of the labeled PE at high $q$ the fast mode dominates, for the labeled PEE the opposite occurs. At lower $q$ under both labeling conditions the weight stays with the slower mode signifying the translational diffusion of the whole molecule. Finally, the RPA-first cumulant which is the respective proper average for the different labeling conditions, is indicated by a dashed and a point dashed line.

As pointed out in Sec. IID the RPA theory predicts two distinct relaxation rates for the PE- and the PEE-labeled materials. In both cases the dynamics of the respective homopolymers should be observed at high $q$ in the Rouse regime. While the experiment shows that the predicted $q$ dependencies are reproduced well by the data, the absolute values for the observed relaxation rates disagree with the predictions (see Table II). In particular the observed Rouse factors for $\mathrm{PE}$ are considerably smaller than predicted, $\left(W l^{4}\right)_{\text {expt }}=2 \times 10^{13} \AA^{4} \mathrm{~s}^{-1}$ compared to $W l_{\mathrm{RPA}}^{4}=3.8$ $\times 10^{13} \AA^{4} \mathrm{~s}^{-1}$ at $T=473 \mathrm{~K}$. At low $q$ values, the two blocks display the same single chain dynamics.

The single chain dynamic structure factor for a whole copolymer chain was measured on sample V containing $10 \%$ of hPEP-hPEE in dPEP-dPEE. In this case, the relaxation of both blocks of the copolymer contributes to the observed spectra. The evaluation proceeded using the procedure already applied for the previous systems. The time regime to determine the initial decay was restricted to $t<10 \mathrm{~ns}$. In Fig. 10(a) the thus obtained experimental values for the first cumulant are compared with the RPA predictions. As may be seen, we obtain a good agreement between predictions and the experimental results. From Fig. 10(b), which presents the weights of the different relaxation modes in the spectra, we observe that the dynamics of a fully labeled chain according to the RPA theory results from an average of the dynamics of the two homopolymers. At large $q$ the initial slope for a fully labeled dilute chain is predicted to coincide with that for the collective dynamics represented by the eigenvalue $\lambda_{3}$.

\section{Collective dynamics}

The collective dynamics of both copolymer systems was probed on the partially labeled copolymer chains hPE-dPEE and dPEP-hPEE. Figure 7(c) presents as an example spectra obtained from the hPE-dPEE system (sample IV) at $T$ $=473 \mathrm{~K}$. As before, the relaxation rate was determined in the initial decay regime $(t<10 \mathrm{~ns})$ by fitting the Rouse dynamic structure factor to the data. Figure 11 compares the thus obtained initial slopes with the RPA predictions for the twocomponent system.

In this case, the RPA predicts that the collective mode $\lambda_{3}$ is the only mode that contributes. At large $q$ good agreement between experiment and RPA prediction is found. Here the first cumulant is proportional to $q^{4}$-we are in the Rouse regime. However, at lower $q$ values $\left(q<0.06 \AA^{-1}\right)$, in the $q$ range where the static structure factor for this block copolymer system has its maximum, significant deviations from the RPA results are obvious. The relaxation rates bend into a nearly $q$-independent plateau regime already around $q$
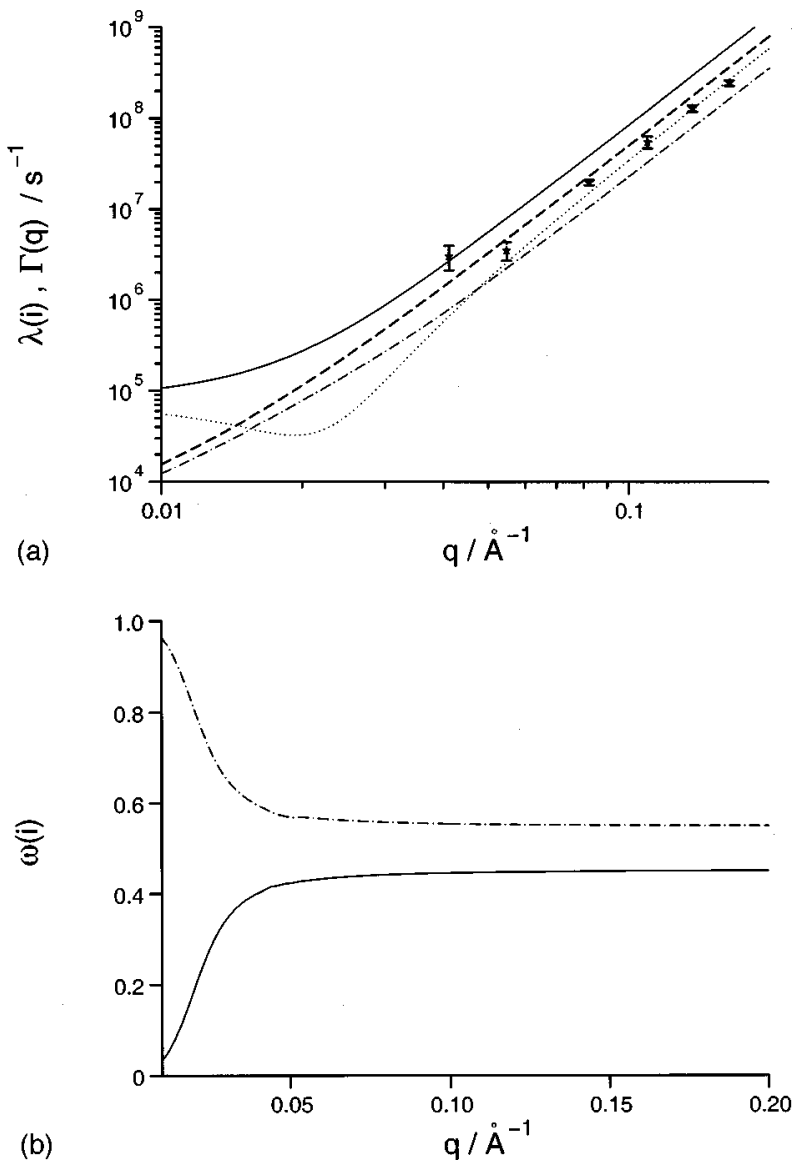

FIG. 10. (a) Rouse relaxation rates (closed circle) from the mixture of $10 \%$ of hPEP-hPEE diblock in a dPEP-dPEE diblock copolymer matrix (sample V) at $473 \mathrm{~K}$. The various lines represent eigenvalues derived from the fourcomponent dynamic RPA approach: $\lambda_{1}$ (solid line), $\lambda_{2}$ (dash-dotted line), $\lambda_{3}$ (dotted line). (b) Relative weights of the eigenvalues plotted in (a). $\omega_{1}$ (solid line), $\omega_{2}$ (dash-dotted line), $\omega_{3}$ (dotted line). The first cumulant is represented by the dashed line in (a).

$=0.06 \AA^{-1}$ and are three to four times faster than predicted. This fast process is not accounted for by the breathing mode of RPA.

Similar observations are made on the higher molecular weight PEP-PEE diblock which undergoes an order-disorder

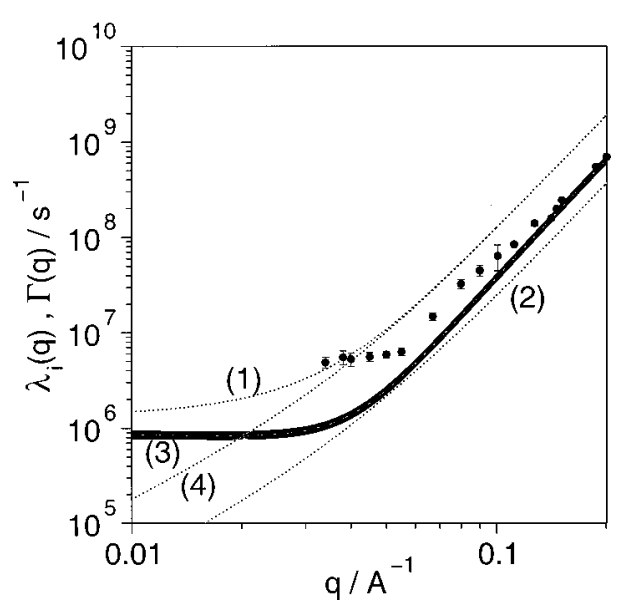

FIG. 11. Characteristic frequencies $\Gamma_{1}(q)$ at $473 \mathrm{~K}$ from the hPE-dPEE melt. 


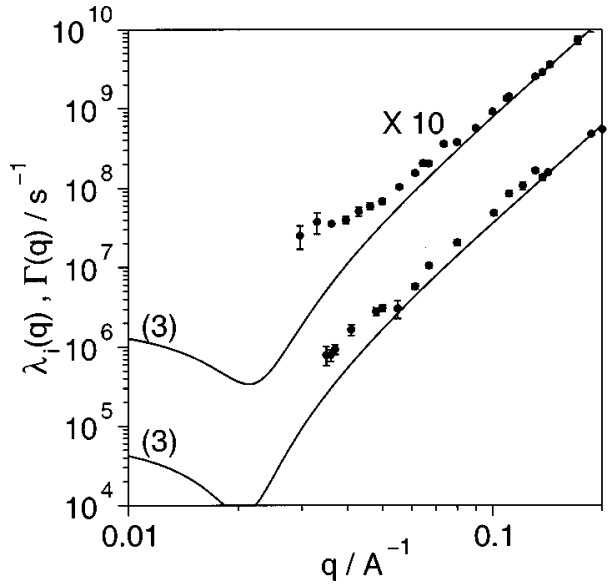

FIG. 12. Characteristic frequencies from the long chain diblock (dPEP$\mathrm{hPEE}$ ) at $533 \mathrm{~K}$ (upper data) and $473 \mathrm{~K}$ (lower data). The solid lines represent the RPA predictions for the collective mode.

transition at $473 \mathrm{~K}$. Figure 12 presents the initial slopes obtained at 473 and $533 \mathrm{~K}$. The RPA predicted collective mode was calculated on the basis of the effective Flory-Huggins parameter and structural parameters taken from the SANS experiment. The friction coefficients again were taken from the experiments on the corresponding homopolymers. At high $q$ there is again good agreement between the measurements and the RPA predictions. But in the low $q$-regime severe deviations from the RPA values are found.

Again these deviations are observed in the peak region of the static structure factor revealing a much faster relaxation of concentrationlike fluctuations than obtained theoretically. The observed faster relaxations slow down in the neighborhood of the order-disorder transition but remain faster than those predicted by the theory.

\section{DISCUSSION}

\section{A. Rouse regime $\left(g R_{g} \gg 1\right)$}

We would like to reiterate that the RPA calculations which we use to compare with our data do not contain any adjustable parameter. The structural information is taken from the SANS experiments, while the chain dynamics is introduced via the Rouse relaxation of the corresponding homopolymers. For all systems considered the associated monomeric friction coefficients have been obtained experimentally by separate NSE studies in the Rouse regime of the respective homopolymer melts.

In the high $q$ regime under all labeling conditions the experimental relaxation rates follow a $q^{4}$ power law-we are in the Rouse regime of the RPA theory. Thus far experiment and theory agree qualitatively. The absolute values for the relaxation rates, however, do not always follow the theoretical predictions. Let us first consider the case of the collective dynamics. Figures 10 and 12 show the quantitative agreement between experiment and RPA prediction in the high $q$ regime for the hPE-dPEE and dPEP-hPEE diblock copolymers. Under this labeling the experiment is sensitive to the collective dynamics (or the concentration fluctuations). From Eq. (36) an observation of the average friction coeffi- cient $\bar{\zeta}$ is predicted. This may also be traced back directly to the equation for the first cumulant [Eq. (33)] which for a visible A component reads

$$
\Gamma(q)=q^{2} k_{B} T m_{a a} / S_{a a}(q) .
$$

In the high $q$-regime $S_{a a}$ is proportional to $\phi_{a} / q^{2}, \phi_{a}$ being the volume fraction of the A component leading immediately to the $q^{4}$-Rouse relaxation.

The mobility factor $m_{a a}$ as it is calculated by the RPA theory, corresponds to the average between the mobility of the homopolymer A, $m_{a a}^{0}$ and the mobility of the surrounding matrix formed by the other components in the mixture denoted by $m_{\mathrm{SM}}^{0}$ with $m_{\mathrm{SM}}^{0}=\Sigma m_{i i}^{0}$ for $i \neq a$,

$$
\frac{1}{m_{a a}}=\frac{1}{m_{a a}^{0}}+\frac{1}{m_{\mathrm{SM}}^{0}} .
$$

For a hA-dB copolymer system, $m_{a a}$ corresponds to the mobility factor which describes the collective dynamics (cd) of the mixture, it is equal to: $\left(m_{\mathrm{cd}}\right)^{-1}=\left(m_{a a}^{0}\right)^{-1}+\left(m_{b b}^{0}\right)^{-1}$. The collective dynamics of diblock chains thus is characterized by an average friction coefficient $\zeta_{\text {cd }}$,

$$
\frac{1}{\zeta_{\mathrm{cd}}}=\frac{1}{f \zeta_{a}+(1-f) \zeta_{b}}
$$

a result we have also found from the eigenvalue $\Gamma_{1}$.

We note that the averaging of the friction coefficients according to Eq. (47) differs from the rule obtained for polymer blends by viscosity measurements. ${ }^{32}$ From these measurements it is inferred that an averaging of the inverse friction coefficients should hold

$$
\frac{1}{\zeta_{\mathrm{cd}}}=\frac{f}{\zeta_{a}}+\frac{1-f}{\zeta_{b}} \text {. }
$$

Following Eq. (48) we would obtain a Rouse factor of $W l^{4}=5 \times 10^{13} \AA^{-4} \mathrm{~s}^{-1}$ for hPE-dPEE $(533 \mathrm{~K})$ instead of $3.45 \times 10^{13} \AA^{-1}$ from Eq. (46) respectively, 3.5 $\times 10^{13} \AA^{-4} \mathrm{~s}^{-1}$ from the experiment. For the PEP-PEE copolymer the corresponding values are $2.9 \times 10^{-13} \AA^{4} \mathrm{~s}^{-1}$ [Eq. (47)], $3.3 \times 10^{-13} \AA \mathrm{s}^{-1}$ [Eq. (46)], and 3 $\times 10^{-13} \AA \mathrm{s}^{-1}$ (expt). In particular for PE-PEE the Rouse factor predicted by Eq. (47) would be significantly higher than measured. Moreover, we observe that the collective Rouse factor of the PE-PEE copolymer is not very different from that for the PEP-PEE system in spite the 2 times faster dynamics of the PE homopolymer in comparison with the PEE homopolymer. This is well described by Eq. (47) while the mixing rule of Eq. (48) fails.

For the contrast situations of samples II and III, which were aiming at the single chain dynamics of the PE and the PEE components, respectively, the same average in the RPA prediction for $m_{a a}$ is performed between the mobility of the labeled component (hPE or hPEE) and that of the surrounding matrix. However, for the collective dynamics the contributions from $\left(m_{a a}^{0}\right)^{-1}$ and $\left(m_{\mathrm{SM}}^{0}\right)^{-1}$ are of the same order leading to an average mobility distinct from that of $m_{a a}^{0}$. In samples II and III only a small labeled fraction is present. Since $m_{a a}^{0}$ is proportional to the A volume fraction, it dominates the inverse sum in this case and the mobility $m_{a a}$ will 
be close-and in the limit of very small volume fractionidentical to $m_{a a}^{0}$. In this limit the first cumulant becomes equal to that of the homopolymer A. Thus, even for a system in which two components $\mathrm{A}$ and $\mathrm{B}$ exhibit strongly different friction coefficients, the RPA theory will predict a relaxation rate close to that of the labeled homopolymer for the single chain dynamics. This result is counterintuitive and obviously spurious but it is related deeply to the basic assumption of dynamic RPA namely to the fact that the bare mobility matrix is diagonal and does not allow, e.g., for off-diagonal elements describing, e.g., mutual friction in the bare system.

Also the values of the diagonal elements of $\underline{\underline{\mu}}^{0}$ might be different from those corresponding to the Rouse rates of the pure homopolymers. Within the RPA framework the bare systems are assumed to be mixed already, however, without any mutual interaction as expressed by the $\kappa$ parameters, the changes that occur in the real system are a consequence of a linear response to the switching on of the $\kappa$ parameters. However, nothing equivalent is provided for the friction terms-which are inherently difficult because friction cannot be cast into an interaction potential. The bare mobility matrix $\underline{\underline{\mu}}^{0}$ completely ignores that the friction-even of a mixture with all $\kappa$ parameters equal to 0 - may be changed due to a different embedding.

Since it is expected that any such modification of the monomeric friction coefficient should depend on the local composition it is not at all clear whether a modification of the ansatz of $\mu^{0}$ would suffice. There are also no rules available that would help to guide how to modify $\mu^{0}$.

The experiments on the PE-PEE system revealed a difference between the single chain dynamics of the PE and the PEE blocks. But the difference is significantly smaller than that predicted by RPA. In particular, the dynamics of the PE block is strongly slowed down by the slow dynamics of the PEE monomers. As discussed above this slowing down of the PE single chain dynamics is not predicted by RPA. On the other hand the single chain dynamics of the PEE component is only slightly accelerated by the presence of the PE monomers and stay rather close to the dynamics of the PEE homopolymer. Thus, other than RPA predicts the single chain dynamics of the two components making up the diblock is affected by the presence of the other component. This effect is stronger for the fast component than for the slower one.

The dynamical RPA theory assumes that all the structural and thermodynamical constraints acting on one component and then on its mobility are described by the static structure factor $S(q)$. Though we observe a good agreement between experiments and RPA predictions for the static properties of a diblock copolymer in the mean field regime, our experiments show that there are dynamical interactions which influence the mobility of components in a polymer mixture. They are not taken into account in the dynamical RPA theory and require an extension of the theory.

\section{B. Low $q$ regime}

At low $q\left(q R_{g} \cong 1\right)$ the ordering effects in block copolymers show their presence: At $q^{*}$ an intensity maximum evolves relating to the formation of lamellar domains in our high $M_{w}$ material. The SANS results on PEP-PEE show the existence of secondary maxima as well as an intensity shoulder above the $1 / q^{2}$ tail of high $q$. Both phenomena cannot be explained in terms of the linear response theory and indicate the presence of well-defined domains beyond simple concentration waves. On the other hand for the short chain material (PE-PEE) where values of only $\kappa N \approx 6-7$ are reached the RPA theory allows an excellent description of all measured SANS patterns.

In this $q$ regime the dynamic RPA predicts the existence of breathing modes for small values of $\kappa N$, where the two blocks move with respect to the common center of mass. With increasing $\kappa N$ important concentration fluctuations evolve, which in the neighborhood of $q^{*}$ should-according to the RPA prediction-induce a significant critical slowing down of these collective modes.

First we consider the short chain PE-PEE diblock for which in the temperature range investigated $\kappa N$ assumes values between 6 and 7. Under these circumstances concentration fluctuations should be weak and in the low $q$ regime under $\mathrm{h}-\mathrm{d}$ labeling we should be confronted with the predicted breathing mode, i.e., the $q$-dependent relaxation rate should become constant around $q R_{g} \cong 2$ (for $R_{g}=49 \AA$ this is expected around $q \approx 0.04 \AA^{-1}$; see Fig. 11). Experimentally, deviations from the Rouse dynamics are observed to commence at $q$ values as high as $q R_{g} \cong 5$ and at $q R_{g}=3$ a crossover to a virtually $q$-independent relaxation rate about four to five times faster than the predicted breathing mode is found. Furthermore, this phenomenon is only visible under $\mathrm{h}-\mathrm{d}$ labeling. Under single chain contrast (Figs. 9 and 10) these deviations from RPA are not seen. Thus, the observed fast relaxation mode must be associated with concentration fluctuations. If we relate the points in $q$, where the deviations start to occur, with length scales, then we conclude that we have found concentration fluctuations at length scales considerably, i.e., up to three times shorter, than predicted by the dynamic RPA theory.

Similarly the results on dPEP-hPEE exhibit deviations from the RPA-Rouse regime already around $q R_{g} \cong 7$ ( $T$ $=533 \mathrm{~K})$. Thereby, the experimentally observed rates differ from the predicted rates up to more than one order magnitude, see Fig. 12. At the phase transition temperature $\left(T_{\mathrm{ODT}}=473 \mathrm{~K}\right)$ however, the effect is greatly reduced and a general slowing down of the fluctuations is found, though some deviations (factor of 2) persist.

Such a behavior would be consistent with an extra scattering contribution on top of the scattering described by the RPA. This extra scattering could account for the fast relaxation and if its intensity is insensitive to the ODT the effect on $\Gamma_{c d}$ would be diminished on approach of the ODT. The "genuine" RPA scattering intensity around $q *$ increases and thereby gets more weight on the initial decay.

The source of the fast extra scattering is not obvious. However, the indication of second-order correlation peaks in the SANS data from PEP-PEE even above the ODT means that concentration profiles in the sample are steeper than simply sinusoidal function, i.e., the existence of some kind of interfaces between $\mathrm{A}$ and $\mathrm{B}$ rich regions may be interfered. ${ }^{33}$ 
These interfaces will fluctuate and thereby yield dynamical scattering contributions, that are not contained in the RPA description. Whether this tentative explanation also may hold for the short chain system PE-PEE is not yet known since the SANS data contain no obvious signature for spatial density variation beyond RPA describability.

\section{Prospects and limitations}

The lowest wave vectors $q$ of the NSE experiments are still somewhat larger than $q^{*}$ of the block copolymers. The region where the theory predicts the biggest effect is only approached from above but not covered. This experimental limitation is not primarily caused by an insufficient $q$ resolution but rather a result of limitations in detectability of minor echo amplitude drops, i.e., $\Delta \cong(S(Q, 0)-S(Q, t$ $\left.\left.=\tau_{e}\right)\right) / S(Q)$. The time range for the determination of initial slopes is limited by the entanglement time $\tau_{e} \cong 10 \mathrm{~ns}$ and not by the time window of the NSE instrument. However, since the initial slope goes down proportional to $q^{-4}$ with high $q$ range and to $q^{-2}$ for $q \rightarrow 0$ the $\Delta$ values to be resolved in the low $q$ regime are not more than a few percent. The limitation therefore stems from statistical counting errors and instabilities of the instrument.

To achieve a better coverage of the $q^{*}$ region a polymer system with a larger $\chi$ parameter is required such that the ODT occurs already at lower molecular weights. In addition the ODT temperature should be high enough to yield sufficiently high mobility values.

\section{CONCLUSION}

The present study is the first detailed test of the dynamical RPA theory in a copolymer melt. Measurements of $S_{i j}(q, t)$ for A-B copolymers melts have been performed around and above the ODT. By different $\mathrm{h}-\mathrm{d}$ labeling single components of $S_{i j}$, different linear combinations (yielding different decay modes) could be observed and be quantitatively compared to the predictions of the state of the art dynamical RPA theory (Akcasu ${ }^{15}$ ).

In the high- $q$ regime a Rouse-type dynamics is observed, RPA derived mixing rules for mobilities (i.e., monomeric friction coefficients) seem to be consistent with the experiment for $100 \% \mathrm{hA}-\mathrm{dB}$ samples exhibiting the collective dynamics. However, the same rules fail for the single chain dynamics as observed in $20 \% \mathrm{hA}-\mathrm{dB}, 80 \% \mathrm{dA}-\mathrm{dB}$ type labeling situation.

At lower $q$ the RPA predicts a slowing down of the collective dynamics around $q^{*}$ near the ODT, whereas the experimentally observed effective initial slopes stay at a much faster level. Obviously some density fluctuation not contained within the RPA contributes to the scattering and leads to a faster dynamics than the RPA at low $q$. A possible source of this scattering could be surface fluctuations.
The deviation found for the mobility combination rules point to some inconsistencies built into the dynamical RPA by the assumption of a bare system mobility matrix containing only diagonal elements, the values of which are identical to those of the pure components.

In addition the extra, fast fluctuation in the collective dynamics observed on approaching $q^{*}$ from the large $q$ side probably indicate the presence of effects due to the violation of the conditions for linear response.

${ }^{1}$ C. B. Muratov, Phys. Rev. Lett. 78, 3149 (1997).

${ }^{2}$ K. A. Koppi, M. Tirrel, and F. S. Bates, Phys. Rev. Lett. 70, 1449 (1993).

${ }^{3}$ M. Sikka, N. Singh, A. Karim, F. S. Bates, S. K. Satija, and C. Majkrzak, Phys. Rev. Lett. 70, 307 (1993).

${ }^{4}$ D. Schwahn, H. Frielinghaus, K. Mortenson, and K. Almdal, Phys. Rev. Lett. 77, 3153 (1996).

${ }^{5}$ L. Leibler, Macromolecules 13, 1602 (1980).

${ }^{6}$ M. Laradji et al., Phys. Rev. Lett. 78, 2577 (1997).

${ }^{7}$ M. Laradji, A. C. Shi, R. Desai, and J. Noolandi, Macromolecules 30, 3242 (1997).

${ }^{8}$ A. M. Mayes and M. Olvera de la Cruz, J. Chem. Phys. 95, 4670 (1991).

${ }^{9}$ J. L. Barrat and G. H. Fredrickson, J. Chem. Phys. 95, 1281 (1991).

${ }^{10}$ G. H. Fredrickson and E. Helfand, J. Chem. Phys. 87, 697 (1987).

${ }^{11}$ M. Gwenza and K. S. Schweizer, J. Chem. Phys. 108, 1257 (1998); 108, 1271 (1998).

${ }^{12}$ U. Genz and T. A. Vilgis, J. Chem. Phys. 101, 7111 (1994).

${ }^{13}$ A. Hoffmann, J. U. Sommer, and A. Blumen, J. Chem. Phys. 106, 6709 (1997); J. Phys. A 30, 5007 (1997).

${ }^{14}$ T. Pakula et al., Macromolecules 30, 8463 (1997).

${ }^{15}$ A. Z. Akcasu et al., Dynamic Light Scattering, the Method and Some Applications (Clarendon, Oxford, 1993).

${ }^{16}$ B. Stühn and A. R. Rennie, Macromolecules 22, 2460 (1989).

${ }^{17}$ M. Tombakoglu and A. Z. Akcasu, Polymer 33, 1127 (1992).

${ }^{18}$ R. Borsali, M. Duval, and M. Benmouna, Polymer 30, 610 (1989); R. Borsali, H. Benoit, J.-F. Legrand, M. Duval, C. Picot, M. Benmouna, and B. Farago, Macromolecules 22, 4119 (1989); R. Borsali, E. W. Fischer, and M. Benmouna, Phys. Rev. A 43, 5732 (1991).

${ }^{19}$ N. Boudenne, S. H. Anastasidadis, G. Fytas, M. Xenidou, N. Hadjichristidis, A. N. Semenov, and G. Fleischner, Phys. Rev. Lett. 77, 506 (1996).

${ }^{20}$ S. H. Anastasiadis, G. Fytas, S. Vogt, and E. W. Fischer, Phys. Rev. Lett. 70, 2415 (1993)

${ }^{21}$ A. N. Semenov, S. H. Anastasiadis, N. Boudenne, G. Fytas, M. Xenidou, and N. Hadjichristidis, Macromolecules 30, 6280 (1997).

${ }^{22}$ A. Hoffmann, T. Koch, and B. Stühn, Macromolecules 26, 7288 (1993).

${ }^{23}$ T. P. Lodge and M. C. Dalvi, Phys. Rev. Lett. 75, 657 (1995).

${ }^{24}$ M. Dalvi, C. E. Eastman, and T. P. Lodge, Phys. Rev. Lett. 71, 2591 (1993).

${ }^{25}$ J. P. Hansen and I. R. McDonald, Theory of Simple Liquids (Academic, New York, 1976).

${ }^{26}$ P. G. De Gennes, Physics (Long Island City, N.Y.) 3, 37 (1967); M. Doi and S. F. Edwards, The Theory of Polymer Dynamics (Clarendon, Oxford, 1986).

${ }^{27}$ M. D. Gehlsen and F. S. Bates, Macromolecules 26, 4122 (1983).

${ }^{28} \mathrm{M}$. Monkenbusch, R. Schätzler, and D. Richter, Nucl. Instrum. Methods Phys. Res. A 399, 301 (1997).

${ }^{29}$ L. J. Fetters, D. J. Lohse, D. Richter, T. A. Witten, and A. Zirkel, Macromolecules 27, 4639 (1994).

${ }^{30}$ J. H. Rosedale, F. Bates, K. Almdal, K. Mortenson, and G. D. Wignall, Macromolecules 28, 1429 (1995).

${ }^{31}$ D. Richter, B. Farago, B. Butera, L. J. Fetters, J. S. Huang, B. Ewen, T. A. Witten, and A. Zirkel, Macromolecules 26, 795 (1993).

${ }^{32}$ G. Meier, G. Fytas, B. Monper, and G. Fleischer, Macromolecules 26, 5310 (1993).

${ }^{33}$ T. Hashimoto, Ta. Koga, Ts. Koga, N. Sakamoto (unpublished). 